University of Zurich

Department of Economics

Working Paper Series

ISSN 1664-7041 (print)

ISSN 1664-705X (online)

Working Paper No. 321

\title{
Testing the Binomial Fixed Effects Logit Model; with an Application to Female Labor Supply
}

Rainer Winkelmann and Lin Xu

Revised version, October 2019 


\title{
Testing the binomial fixed effects logit model; with an application to female labor supply*
}

\author{
RAINER WINKELMANN \\ University of Zurich
}

\author{
LIN XU \\ University of Zurich
}

October 2019

\begin{abstract}
Regression models for proportions are frequently encountered in applied work. The conditional expectation function is bounded between 0 and 1 and therefore must be non-linear, requiring nonstandard panel data extensions. One possible approach is the binomial panel logit model with fixed effects (Machado, 2004). We propose a new and simple implementation of the conditional maximum likelihood estimator for standard software. We investigate the properties of the estimator under misspecification and derive a new test for overdispersion. Estimator and test are applied in a study of contracted working volumes, measured as proportion of full-time work, for women in Switzerland.
\end{abstract}

Keywords: proportions data, unobserved heterogeneity, conditional maximum likelihood, overdispersion

JEL classification: C23, J21.

\footnotetext{
* Department of Economics, University of Zurich, E-mail: rainer.winkelmann@econ.uzh.ch and lin.xu@econ.uzh.ch. We are grateful to Damian Kozbur, Johannes Kunz, Kevin Staub and Tiemen Woutersen for helpful discussions. The data used in the application have been provided by the Swiss Household Panel. We gratefully acknowledge financial support by the Swiss National Science Foundation.
} 


\section{TITLEPAGE}

\section{Introduction}

After half a century of research on econometric models for limited dependent variables (Maddala, 1983, Wooldridge, 2002), it remains the case that only a small portion of it deals with proportions data, and even a smaller one with panel models for such proportions. Machado (2004) proposes the binomial fixed effects logit model, Papke and Wooldridge (2008) a correlated random effects probit quasi-likelihood estimator and Ramalho et al. (2016) a class of exponential GMM estimators.

And yet, proportions and related types of data are regularly encountered in applied econometric work. Often, they correspond to the number of "successes" in a sequence of Bernoulli trials, such as homicide- or unemployment rates, or the fraction of days absent from work during a work week. Also, variety scores (e.g., the number of applicable items in a general health questionnaire), bounded count data, as well as ratings, share the key features of such variables, discreteness and the existence of an upper and lower bound for the outcome.

In all these cases, the binomial model with a logit function for the expected proportion provides a natural starting point for modelling. For the fixed effects setting, Machado (2004) shows that the incidental parameters problem can be overcome by a conditional maximum likelihood (CML) estimator, much like it is the case for the binary response logit model (Chamberlain, 1980). She also provides Monte Carlo evidence indicating that the dummy variables (DV) approach is subject to an upward bias that is decreasing both in the length of the panel, $T$, and in the number of Bernoulli trials, $K$. For $T>5$ and $K>5$, CML and DV approaches yield quite comparable results with minor bias (Machado, 2004).

This paper advances the earlier literature in three directions: First, we show how the binomial logit fixed effects estimator can be implemented in any off-the-shelf statistical software that includes a conditional logit routine, using the idea of cloning, or data expansion. Second, we study the properties of the CML and DV estimators for the case where the binomial distributional assumption fails. The leading example is that of overdispersion, originating from unobserved heterogeneity or dependence among the Bernoulli trials. The CML estimator is not a pseudo ML estimator in the sense of Gourieroux, Monfort and Trognon (1984), and it does not possess formal robustness properties. We therefore investigate the extent of bias in a series of simulation experiments. Third 
and finally, we derive and implement a new test for the binomial assumption, i.e., a test for the hypothesis of no dispersion, as existing tests (e.g. Dean, 1992) cannot be applied because the fixed effects are not estimated by the CML estimator.

To illustrate the proposed methods, we conduct a study of the determinants of women's work behavior in Switzerland. The outcome variable is the contracted work-time percentage, where 0 means no work and 1 means full-time work. Data are from the Swiss Household Panel for the years 2012 to 2016. The binomial logit estimates indicate that having children is associated with substantially reduced work-time percentage, ceteris paribus. Perhaps more surprisingly, having a partner makes the effect more pronounced, whereas speaking French reduces it.

\section{Model and estimation}

A proper panel model for proportions $y_{i t} \in[0,1]$ must overcome two challenges. First, the model should observe the restricted support of the outcome, as well as being able to handle data clustering at the end points. For instance, the log-odds transformation $\log \left[y_{i t} /\left(1-y_{i t}\right)\right]$ is not defined for $y_{i t}=0$ or $y_{i t}=1$. Another method facing the same limitation is beta regression, which is flexible for fitting continuous proportional data but cannot give predictions at the boundaries with positive probability. Second, direct control for unobserved time-invariant individual heterogeneity (that may or may not be correlated with the regressors) using a dummy for each cross-sectional unit is subject to the incidental parameters problem, leading to inconsistent estimation of structural parameters when the length of panel $T$ is fixed.

Machado (2004) addresses these two issues by introducing the binomial fixed effects logit model for proportions and proposing a consistent conditional maximum likelihood estimator as follows:

\section{Assumption 1}

Let $Y_{i t}=K y_{i t}$, where $K$ is a known integer and

$$
y_{i t} \in\left\{0, \frac{1}{K}, \frac{2}{K}, \ldots, 1\right\}
$$

such that

$$
Y_{i t} \mid p_{i t} \sim \operatorname{binomial}\left(K, p_{i t}\right), \quad i=1, \ldots, N ; \quad t=1, \ldots, T
$$


Here, $K$ is the number of "trials", $Y_{i t}=K y_{i t}$ is the "number of successes", and $y_{i t}$ is the proportion, or fraction of successes for observation unit $i$ in period $t$.

\section{Assumption 2}

Let the expected proportion depend on covariates $x_{i t}$ and an individual specific effect $\alpha_{i}$ as follows:

$$
\mathrm{E}\left(y_{i t} \mid x_{i t}, \alpha_{i}\right)=p_{i t}=\frac{\exp \left(x_{i t}^{\prime} \beta+\alpha_{i}\right)}{1+\exp \left(x_{i t}^{\prime} \beta+\alpha_{i}\right)} \equiv \Lambda_{i t}
$$

$x_{i t}$ and $\alpha_{i}$ can be correlated.

\section{Assumption 3}

Observations are independent between individuals and, conditional on group effects $\alpha_{i}$, serially uncorrelated.

The objective of the analysis is estimation of $\beta$. Under Assumptions 1-3, the joint binomial density for $Y_{i 1}, Y_{i 2}, \ldots, Y_{i T}$ conditional on $\sum_{t} Y_{i t}$ is given by (see Machado, 2004)

$$
f\left(Y_{i 1}, Y_{i 2}, \ldots, Y_{i T} \mid \sum_{t} Y_{i t}\right)=\frac{\Pi_{t}\left(\begin{array}{c}
K \\
y_{i t}
\end{array}\right) p_{i t}^{Y_{i t}}\left(1-p_{i t}\right)^{K-Y_{i t}}}{\sum_{q \in Q_{i}} \Pi_{t}\left(\begin{array}{c}
K \\
q_{t}
\end{array}\right) p_{i t}^{q_{t}}\left(1-p_{i t}\right)^{K-q_{t}}}=\frac{\exp \left(\sum_{t} Y_{i t} x_{i t}^{\prime} \beta\right) \Pi_{t}\left(\begin{array}{c}
K \\
y_{i t}
\end{array}\right)}{\sum_{q \in Q_{i}} \exp \left(\sum_{t} q_{t} x_{i t}^{\prime} \beta\right) \Pi_{t}\left(\begin{array}{c}
K \\
q_{t}
\end{array}\right)}
$$

where $Q_{i}=\left\{\left(q_{1}, q_{2}, \ldots, q_{T}\right) \mid q_{t} \in\{0,1,2, \ldots, K\}, \sum_{t} q_{t}=\sum_{t} Y_{i t}\right\}$. The conditional binomial approach eliminates the fixed effects $\alpha_{i}$ which appear in the numerator and denominator with same power. Observations for which $\sum_{t} Y_{i t}=0$ or $\sum_{t} Y_{i t}=K T$ have a conditional probability of 1 and do not contribute to estimation of $\beta$. For proportion data, such outcomes tend to be much less prevalent than for binary outcomes.

\subsection{An alternative implementation}

The binomial logit fixed effects estimator can be implemented using any off-the-shelf statistical software with a conditional logit routine, since the binomial distribution arises as the sum of $K$ independent Bernoulli trials. Therefore, two estimators are equivalent: one based on a binomial log-likelihood function and the other based on a Bernoulli log-likelihood for an expanded dataset.

For the expanded dataset, one simply generates a sequence of $K$ copies for each $i$, keeping the 
regressors unchanged, where the proportion $y_{i t}$ is replaced by a sequence of $0 / 1$ indicator variables $d_{i j t}$ in arbitrary order such that

$$
\sum_{j=1}^{K} d_{i j t}=K y_{i t}
$$

It follows that $d_{i j t}$ and $y_{i t}$ have the same CEF:

$$
\mathrm{E}\left(y_{i t} \mid x_{i t}\right)=\mathrm{E}\left(\frac{\sum_{j=1}^{K} d_{i j t}}{K} \mid x_{i t}\right)=\mathrm{E}\left(d_{i j t} \mid x_{i t}\right)
$$

The logit (Bernoulli) log-likelihood function of the expanded dataset is given by

$$
\begin{aligned}
\log L & =\sum_{i}^{N} \sum_{j}^{K} d_{i j t} \log \left(\Lambda_{i t}\right)+\left(1-d_{i j t}\right) \log \left(1-\Lambda_{i t}\right) \\
& =\sum_{i}^{N} Y_{i t} \log \left(\Lambda_{i t}\right)+\left(K-Y_{i t}\right) \log \left(1-\Lambda_{i t}\right) .
\end{aligned}
$$

This log-likelihood function is proportional to the binomial log-likelihood as well as to the Bernoulli quasi-log-likelihood (Papke and Wooldridge, 1996, replacing $Y_{i t}$ by $y_{i t}$ and $\left(K-Y_{i t}\right)$ by $\left(1-y_{i t}\right)$ ), and the three ML estimators are therefore identical.

Similarly, the conditional density function for individual $i$ at time $t$ can be written as:

$$
f\left(\left\{d_{i j t}\right\} \mid \sum_{t} \sum_{j} d_{i j t}\right)=\frac{\Pi_{t} \Pi_{j} p_{i t}^{d_{i j t}}\left(1-p_{i t}\right)^{1-d_{i j t}}}{\sum_{s \in S_{i}} \Pi_{t} \Pi_{j} p_{i t}^{s_{j t}}\left(1-p_{i t}\right)^{1-s_{j t}}}=\frac{\exp \left(\sum_{t} \sum_{j} d_{i j t} x_{i t}^{\prime} \beta\right)}{\sum_{s \in S_{i}} \exp \left(\sum_{t} \sum_{j} s_{j t} x_{i t}^{\prime} \beta\right)}
$$

where $\left.S_{i}=\left\{\left(s_{11}, s_{21}, \ldots, s_{K 1}, s_{12}, \ldots, s_{K T}\right) \mid s_{j t} \in\{0,1\}, \sum_{t} \sum_{j} s_{j t}=\sum_{t} \sum_{j} d_{i j t}\right)\right\}$.

Compared with equation (3), the number of $s$ such that $\left\{s \mid \sum_{j} s_{i j t}=q_{i t}\right\}$ is $\left(\begin{array}{l}K \\ q_{i t}\end{array}\right)$ for given $q$. Equation (6) is therefore basically the same as equation (3), except for the term $\Pi_{t}\left(\begin{array}{l}K \\ y_{i t}\end{array}\right)$ in the numerator. But this term does not depend on any parameter and thus drops out of the first-order condition for the maximum of the log-likelihood function. Specifically, the conditional Bernoulli 
log-likelihood function can be written as:

$$
\log L=\sum_{i}\left[\sum_{t} \sum_{j} d_{i j t} x_{i t}^{\prime} \beta-\log \left(\sum_{s \in S_{i}} \exp \left(\sum_{t} \sum_{j} s_{j t} x_{i t}^{\prime} \beta\right)\right)\right]
$$

with first derivative

$$
\frac{\partial \log L}{\partial \beta}=\sum_{i}\left[\sum_{t} K y_{i t} x_{i t}^{\prime}-\frac{\sum_{s \in S_{i}} \exp \left(\sum_{t} \sum_{j} s_{j t} x_{i t}^{\prime} \beta\right) \sum_{t} \sum_{j} s_{j t} x_{i t}^{\prime}}{\sum_{s \in S_{i}} \exp \left(\sum_{t} \sum_{j} s_{j t} x_{i t}^{\prime} \beta\right)}\right]
$$

which is the same as in the conditional binomial model, up to an additive constant, and will give the same consistent estimator of $\beta$, after elimination of the fixed effects.

\subsection{Overdispersion}

Departures from the binomial proportions model can take a number of forms. The first one is a violation of the independence assumption for the underlying Bernoulli trials. Positive dependence, or contagion, among the sequence of Bernoulli trials causes overdispersion, a conditional variance exceeding the binomial variance $K p_{i t}\left(1-p_{i t}\right)$. Another violation is "unobserved heterogeneity", where $p_{i t}$ is no longer a constant but rather a random variable, say $\tilde{p}_{i t}$. Marginalizing over $\tilde{p}_{i t}$ then leads to a mixture model that is characterized by overdispersion as well. Depending on the distribution of $\tilde{p}_{i t}$, proportions can for example have a u-shaped probability function even conditional on $\alpha_{i}$ and $x_{i t}$, i.e., probability mass stacked at the endpoints of 0 and 1 , which is never the case for a binomial distribution that has either a single, or two adjacent modes.

A prominent example for a continuous mixture is the beta-binomial model, where the conditional probability is

$$
\tilde{p}_{i t} \sim \operatorname{beta}\left(u_{i t}, v_{i t}\right)
$$

and

$$
u_{i t}=\phi \Lambda\left(x_{i t} \beta+\alpha_{i}\right), v_{i t}=\phi\left(1-\Lambda\left(x_{i t} \beta+\alpha_{i}\right)\right) .
$$

It is easy to show that a beta-binomial distribution with this parameterization has expectation 
$K \Lambda_{i t}$ and variance

$$
\operatorname{Var}\left(Y_{i t} \mid K, \Lambda_{i t}, \phi\right)=K \Lambda_{i t}\left(1-\Lambda_{i t}\right)\left(1+\frac{K-1}{\phi+1}\right)
$$

Thus, the variance of the beta-binomial model is proportional to that of the binomial model. The degree of overdispersion increases in $K$, the number of trials, and it decreases in the parameter $\phi$. The binomial variance is obtained for $K=1$, or in the limit, for $\phi \rightarrow \infty$, which also means that $\operatorname{Var}\left(\tilde{p}_{i t}\right) \rightarrow 0$.

In general, fixed effects conditional maximum likelihood estimators are not consistent if the underlying model is misspecified. The reason is that the first-order condition is not a moment condition for the mean, but rather a function of the conditional probabilities. However, it might still be the case that the CML estimator works satisfactorily as long as the degree of overdispersion, in other words, the departure from the binomial assumption, is not too large. We will explore this type of robustness in a series of simulation experiments. We thereby extend results by Machado (2004), who considered the severity of the incidental parameters problem and the small sample properties of the CML estimator under the maintained assumption of a correctly specified binomial model. In our simulations, this assumption is dropped.

\subsection{Simulation study}

The simulation experiments employ two different data generating processes: one where the binomial assumption is satisfied, and a second one, based on the beta-binomial model, where overdispersion is present. Unobserved time-invariant individual heterogeneity is positively correlated with the regressor in both cases. The degree of overdispersion is varied from 10 to 100 percent.

Both set-ups use the same logit conditional expectation function with a single regressor

$$
\mathrm{E}\left(y_{i t} \mid x_{i t}, \alpha_{i}\right)=\Lambda\left(\beta_{0}+\beta_{1} x_{i t}+\alpha_{i}\right)=\frac{\exp \left(\beta_{0}+\beta_{1} x_{i t}+\alpha_{i}\right)}{1+\exp \left(\beta_{0}+\beta_{1} x_{i t}+\alpha_{i}\right)} \quad,
$$

where $\beta_{0}=0, \beta_{1}=2$ and the size of the cross-section is either $N=100$ or $N=500$. The time dimension increases from $T=2, T=5$ to $T=10$.

The regressor $x_{i t}$ is drawn from a uniform distribution with support $[-1,1]$ and has therefore a mean of 0 and a variance of $1 / 3$. Draws are independent both across individuals and over time. 
We make a correlated random effects assumption:

$$
\alpha_{i}=\sqrt{T} \bar{x}_{i}+\varepsilon_{i}
$$

where $\varepsilon_{i} \sim N(0,1)$. It follows that the correlation between $\alpha_{i}$ and $\bar{x}_{i}$ is 0.5 , a substantial amount. Once the mean is given, the dependent variable is obtained by generating pseudo random numbers from either a binomial or a beta-binomial distribution. Specifically, we first draw integer random numbers from a (beta) binomial distribution with parameters $K$ and $\Lambda\left(x_{i t} \beta_{1}+\alpha_{i}\right)$, and then divide the result by the number of categories $K$. e.g.:

$$
y_{i t}=\frac{K y_{i t}}{K}, K y_{i t} \sim \operatorname{binomial}\left(K, p_{i t}\right), p_{i t}=\Lambda\left(\beta_{0}+\beta_{1} x_{i t}+\alpha_{i}\right)
$$

$K$ is exogenously set to 2,5 or 10 . For $K=2, K \times y_{i t}$ can be 0,1 , or 2 , with corresponding fractions of $y_{i t}=0,0.5$, or 1 , respectively; if $K=10, y_{i t}$ takes on one-digit decimals: $0,0.1,0.2, \ldots$, 1.

Ignoring the presence of the individual specific component and estimating the marginal, pooled model will have two effects:

- $\beta_{1}$ is upward biased due to the positive correlation between $x_{i t}$ and $\alpha_{i}$.

- $\beta_{1}$ is downward biased due to omitted heterogeneity. In the probit model, there is a closed form expression for this bias (Wooldridge, 2002). In the logit model, it needs to be computed numerically, but the direction is the same.

Which one of the two biases is larger is an empirical matter. The DV estimator, on the other hand, suffers from the standard upward incidental parameters bias (Abrevaya, 1997).

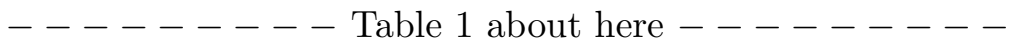

Table 1 shows the simulation results based on 1000 replications, for a sample size of $N=100$. The dependent variable has a binomial distribution conditional on $x_{i t}$ and $\alpha_{i}$, and $K=2, K=5$ or $K=10$. The mean and standard deviation of estimated coefficients across replications are reported. The three estimators are referred to as Blogit CML, Blogit DV, and pooled logit respectively. 
As Machado (2004), we find that the Blogit CML model estimates the true structural slope parameter very well even for small samples. There is a $2 \%$ upward bias for $T=K=2$ that vanishes quickly as either $T$ or $K$ increases. The sampling variability increases not only in $T$ but also in $K$, albeit at a less than $\sqrt{K}$ rate. The Blogit DV estimators have a larger bias and a larger standard error, and hence a higher mean squared error, in all settings. It becomes small as $T$ and $K$ increase. For instance, for $T=10$ and $K=10$, the mean Blogit DV estimate is 2.025, whereas the mean Blogit CML estimate is 2.000. On the other hand, the pooled logit estimator has no tendency to converge to the true parameter $\beta_{1}=2$, over- or underestimating it depending on the DGP. In the lower panel of Table 1, simulations are repeated for a larger sample, $N=500$ instead of $N=100$. The qualitative conclusions remain unchanged.

\section{Beta-binomial DGP}

Simulations from the beta-binomial model add a further step: instead of directly obtaining binomial responses with (conditional on $x_{i t}$ and $\alpha_{i}$ ) success probability $p_{i t}=\Lambda\left(\beta_{0}+\beta_{1} x_{i t}+\alpha_{i}\right), \tilde{p}_{i t}$ is now drawn from a beta distribution with mean $p_{i t}$ :

$$
\tilde{p}_{i t} \sim \operatorname{beta}\left(\phi \Lambda\left(\beta_{0}+\beta_{1} x_{i t}+\alpha_{i}\right), \phi\left(1-\Lambda\left(\beta_{0}+\beta_{1} x_{i t}+\alpha_{i}\right)\right)\right)
$$

From (10), we know that the multiplicative variance inflation factor depends both on $K$ and $\phi$. To keep the degree of overdispersion constant for $K=2,5,10$, we adjust $\phi$ accordingly. For example, for $10 \%$ overdispersion and $K=2$, we have $1+(K-1) /(\phi+1)=1.1$, so $\phi=9$.

As a practical limitation, common beta random number generators set lower bounds (above the theoretical ones of 0) for the two parameters. In Stata, for example, these are given by 0.05 and 0.15, respectively. From (14) we see that attempts to draw from the beta using arguments violating these bounds are more likely to arise when the mean is close to zero or one, or when $\phi$ is small (and therefore the degree of overdispersion is large). Since such occurrences only depend on exogenous factors, dropping these cases does not invalidate the estimation procedure. However, it affects the effective sample size and thus leads to higher standard errors than would otherwise be the case.

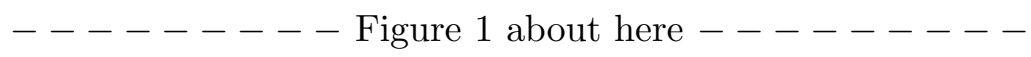

Figures 1 and 2 plot the relative biases of Blogit CML and Blogit DV against the degree of overdispersion, for $N=100$ and $N=500$, respectively. Overdispersion varies from $10 \%$ to $200 \%$ (The full 
results on the means and standard deviations of the estimators for each DGP are given in Tables A1 and A2 in the appendix).

Three key patterns emerge. First, overdispersion leads to an upward bias of both the Blogit CML and the Blogit DV estimators. The bias increases in the amount of overdispersion. Second, the Blogit CML estimator always dominates the Blogit DV estimator, both in terms of bias and standard error. This result did already hold for the binomial case, and it persists in the presence of overdispersion. Third, for a given degree of overdispersion, the bias is decreasing in $T$ as well as in $K$. However, increasing $K$ alone non necessarily leads to a reduction in estimation bias, because it leads to more overdispersion, ceteris paribus. Again, results are qulitatively similar for $N=500$ (see Figure 2).

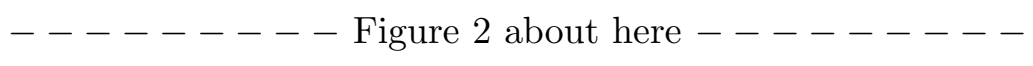

The overall conclusion is that the Blogit CML estimator maintains a rather good performance even if the binomial model is misspecified, as long as the degree of overdispersion is modest, or else, as long as $T$ is large. To take the two polar cases, for $N=100$, if $K=T=2$ the mean estimate with $10 \%$ overdispersion is 2.1 , a $5 \%$ upward bias. For $K=T=10$ the mean estimate with $100 \%$ overdispersion is 2.049 , a $2.45 \%$ upward bias.

\section{A test for overdispersion}

Existing binomial tests for $y_{i t}$, e.g. Dean's (1992) score test or regression based tests regressing squared residuals $y_{i t}-\hat{\Lambda}_{i t}$ on $\hat{\Lambda}_{i t}\left(1-\hat{\Lambda}_{i t}\right.$ ), require estimates $\hat{\Lambda}_{i t}$ (that are needed for estimating the conditional variances $\left.\widehat{\operatorname{Var}}\left(y_{i t} \mid x_{i t}\right)\right)$. However the Blogit CML approach does not give us $\hat{\alpha}_{i}$, so this is not feasible. To ascertain the validity of the Blogit CML model assumption, i.e. that $K y_{i t}$ is binomial distributed conditional on $\alpha_{i}$ and $x_{i t}$, we propose an alternative approach that uses $\hat{\beta}$ but does not require estimates of $\alpha_{i}$, based on taking differences.

To start, consider a binary random variable $M_{i t}$ defined by a draw from a Bernoulli distribution with mean $y_{i t}, M_{i t} \sim \operatorname{Bernoulli}\left(y_{i t}\right)$. Clearly, the conditional mean is $\mathrm{E}\left(M_{i t} \mid y_{i t}\right)=y_{i t}$ while the unconditional mean is $\mathrm{E}\left(M_{i t}\right)=\Lambda_{i t}$. The conditional variance is $\operatorname{Var}\left(M_{i t} \mid y_{i t}\right)=y_{i t}\left(1-y_{i t}\right)$, while the unconditional variance is $\operatorname{Var}\left(M_{i t}\right)=\mathrm{E}\left[y_{i t}\left(1-y_{i t}\right)\right]+\operatorname{Var}\left(y_{i t}\right)=\Lambda_{i t}\left(1-\Lambda_{i t}\right)$.

The basic idea of the test is to compare the variances of the differences $Y_{i t}-Y_{i s}$ and that of the 
difference $M_{i t}-M_{i s}$, for pairs of observations where the underlying probabilities $p_{i t}=\Lambda_{i t}$ are the same (or similar) for the two periods. For notational simplicity, let $t=1$ and $s=2$. In such cases, outcomes $Y_{i 1}, Y_{i 2}$ can be regarded under $H_{0}$ as random draws from i.i.d. binomial distributions and the variance of $Y_{i 1}-Y_{i 2}$ should be equal to the sum of binomial variances, under assumptions A1 and A3. On the other hand, the Bernoulli draws from the same distributions have standard variances. If there is over- or under- dispersion, the variance of $Y_{i 1}-Y_{i 2}$ will be larger or smaller than the variance calculated from Bernoulli draws.

Specifically, consider the variable

$$
z_{i}=\frac{\left(Y_{i 1}-Y_{i 2}\right)^{2}-K\left(M_{i 1}-M_{i 2}\right)^{2}}{K(K-1)} .
$$

Conditional on $y_{i 1}, y_{i 2}$,

$$
\begin{aligned}
\mathrm{E}\left[\left(M_{i 1}-M_{i 2}\right)^{2} \mid y_{i 1}, y_{i 2}\right] & =y_{i 1}\left(1-y_{i 1}\right)+y_{i 2}\left(1-y_{i 2}\right)+\left(y_{i 1}-y_{i 2}\right)^{2} \\
& =y_{i 1}+y_{i 2}-2 y_{i 1} y_{i 2} .
\end{aligned}
$$

Therefore, under $\mathrm{A} 1, \mathrm{~A} 2$ and $\mathrm{A} 3$, the expectation of $z_{i}$ is given by

$$
\mathrm{E}\left(z_{i}\right)=\frac{1}{K(K-1)}\left[\operatorname{Var}\left(Y_{i 1}\right)+\operatorname{Var}\left(Y_{i 2}\right)+\left(\mathrm{E} Y_{i 1}-\mathrm{E} Y_{i 2}\right)^{2}-K\left(\Lambda_{i 1}+\Lambda_{i 2}-2 \Lambda_{i 1} \Lambda_{i 2}\right)\right]
$$

Under the binomial assumption, $\operatorname{Var}\left(Y_{i t}\right)=K \Lambda_{i t}\left(1-\Lambda_{i t}\right)$, and it follows that

$$
\begin{aligned}
\mathrm{E}\left(z_{i}\right)= & \frac{1}{K(K-1)}\left[K \Lambda_{i 1}\left(1-\Lambda_{i 1}\right)+K \Lambda_{i 2}\left(1-\Lambda_{i 2}\right)+K^{2}\left(\Lambda_{i 1}-\Lambda_{i 2}\right)^{2}\right. \\
& \left.-K\left[\Lambda_{i 1}\left(1-\Lambda_{i 1}\right)+\Lambda_{i 2}\left(1-\Lambda_{i 2}\right)+\left(\Lambda_{i 1}-\Lambda_{i 2}\right)^{2}\right]\right] \\
= & \left(\Lambda_{i 1}-\Lambda_{i 2}\right)^{2} .
\end{aligned}
$$

Hence, the expected value of $z_{i}$ is zero under the null hypothesis of binomial dispersion as long as $\Lambda_{i 1}=\Lambda_{i 2}$. From (2), this is the case for observations for which the regressors $x_{i t}$ do not change over time, $x_{i 1}=x_{i 2}$.

Under the alternative hypothesis, the variance function is given by

$$
\operatorname{Var}\left(Y_{i}\right)=K \Lambda_{i t}\left(1-\Lambda_{i t}\right)(1+\eta), \quad \eta \neq 0
$$

For the beta-binomial model, $\eta$ is equal to $\eta=\frac{K-1}{\phi+1}>0$. If $\eta<0, Y_{i}$ is under-dispersed relative to the binomial model. Overdispersion originates from positive dependence of the Bernoulli trials, or 
from unobserved heterogeneity. Underdispersion arises from negative dependence. If $y_{i t}$ does not follow $H_{0}$, the test statistic will have positive sign in overdispersed samples and negative sign for underdispersed ones.

\subsection{Case I: discrete covariates}

Define the set of individuals with the same expectations over time, $A=\left\{i: \Lambda_{i 1}=\Lambda_{i 2}\right\}$, for which $E\left(z_{i} \mid i \in A\right)=0$ holds. With time invariant fixed effect $\alpha_{i}$, the set $A$ is equal to $\left\{i: x_{i 1}=x_{i 2}\right\}$. It is feasible to find such a set $A$ if all covariates are finite discrete variables and assuming that the $x$-values are drawn from a stationary distribution, ruling out pure time trends, for instance. The test term for discrete $x_{i t}$ is defined as

$$
\tau_{A}=\hat{\mathrm{E}}\left(z_{i} \mid i \in A\right)=\frac{\sum_{i \in A} z_{i}}{|A|}
$$

where $|A|$ represents the number of elements in $A$. Under $H_{0}, \tau_{A} \stackrel{p}{\longrightarrow} 0$. Further, by the central limit theorem (CLT), the statistic $\tau_{A}$ converges to a normal distribution,

$$
\sqrt{|A|}\left(\tau_{A}-0\right) \stackrel{d}{\longrightarrow} N\left(0, \sigma_{A}^{2}\right)
$$

where $\sigma_{A}^{2}=\operatorname{Var}\left(z_{i} \mid i \in A\right)$. In practice, $\sigma_{A}^{2}$ is replaced by the sample variance $\hat{\sigma}_{A}^{2}$. So we reject the binomial distribution assumption at the $\alpha \%$ significance level if $\left|\frac{\tau_{A}}{\hat{\sigma}_{A} / \sqrt{|A|}}\right| \geq Z_{1-\frac{\alpha}{2}}$.

Individuals in the set $A$ do not contribute to the estimation of the Blogit CML model, since $x_{i t}$ are cancelled out as fixed effects. Nonetheless, they are needed for generating our dispersion test. This non-parametric method to build a test is similar to finding proper cell estimators in matching theory, but likewise faces the curse of dimensionality. It is hard to find the set $A$ when the dimension of $x_{i t}$ becomes larger. If $|A|$ shrinks, the convergence rate $\sqrt{|A|}$ will decrease and the estimator $\tau_{A}$ will converge more slowly.

\subsection{Case II: continuous covariates}

The set $A=\left\{i: \Lambda_{i 1}=\Lambda_{i 2}\right\}$ is empty or very small when $\Lambda_{i 1}$ and $\Lambda_{i 2}$ are continuous. A more general method uses a kernel estimator for the conditional expectation $\mathrm{E}\left(z_{i} \mid \Lambda_{i 1}-\Lambda_{i 2}=0\right)$. The main idea is to put more weight on individuals with smaller $\left|\Lambda_{i 1}-\Lambda_{i 2}\right|$. Since we do not observe the 
underlying expectations $\Lambda_{i t}$ directly, we find the set $A$ by using observables $x_{i t}$. Under the assumption of a single scalar regressor and time-invariant unobserved heterogeneity, we can decompose the conditional expectation (16) by a Taylor expansion at $x_{i 2}$,

$$
\begin{aligned}
\left(\Lambda_{i 1}-\Lambda_{i 2}\right)^{2} & =\left[\Lambda\left(x_{i 1} \beta+\alpha_{i}\right)-\Lambda\left(x_{i 2} \beta+\alpha_{i}\right)\right]^{2} \\
& =\left[\Lambda^{\prime}\left(x_{i 2} \beta+\alpha_{i}\right) \beta\left(x_{i 1}-x_{i 2}\right)+\frac{\Lambda^{\prime \prime}\left(x_{i 2} \beta+\alpha_{i}\right)}{2 !} \beta^{2}\left(x_{i 1}-x_{i 2}\right)^{2}+o\left(\left(x_{i 1}-x_{i 2}\right)^{2}\right)\right]^{2} \\
& =\left[\Lambda^{\prime}\left(x_{i 2} \beta+\alpha_{i}\right) \beta\left(x_{i 1}-x_{i 2}\right)\right]^{2}+o\left(\beta^{2}\left(x_{i 1}-x_{i 2}\right)^{2}\right),
\end{aligned}
$$

Denote $\Delta_{i}=\left(x_{i 1}-x_{i 2}\right) \beta$,

$$
E\left(z_{i} \mid \Lambda_{i 1}-\Lambda_{i 2}\right)=\left(\Lambda_{i 1}-\Lambda_{i 2}\right)^{2}=\left(\Lambda_{i 2}^{\prime} \Delta_{i}\right)^{2}+o\left(\Delta_{i}^{2}\right)
$$

As the fixed effect $\alpha_{i}$ is cancelled out, an alternative conditional expectation function is given by $\Delta_{i}$,

$$
\tau(\Delta)=E\left(z_{i} \mid \Delta_{i}=\Delta, X_{i}\right)=\left(\Lambda_{i 2}^{\prime} \Delta\right)^{2} .
$$

Then, under the binomial assumption,

$$
E\left(z_{i} \mid \Lambda_{i 1}-\Lambda_{i 2}=0\right)=\tau(0)=0 .
$$

The result generalizes to a vector-valued $x$, in which case $\Delta_{i}=\left(x_{i 1}-x_{i 2}\right)^{\prime} \beta$.

The next step is to build a kernel estimator for $\tau(0)$. One conditional moment estimator for $\tau(\Delta)$ is $\hat{\tau}(\Delta)=\frac{\sum_{i=1}^{N} K\left(\frac{\Delta_{i}-\Delta}{h}\right) z_{i}}{\sum_{i=1}^{N} K\left(\frac{\Delta_{i}-\Delta}{h}\right)}$, where $h$ is the kernel bandwidth for $\Delta_{i}$ and $K\left(\frac{\Delta_{i}-\Delta}{h}\right)$ is the kernel function. For a given sample, $\Delta_{i}$ needs to be replaced by $\hat{\Delta}_{i}=\left(x_{i 1}-x_{i 2}\right) \hat{\beta}$, where $\hat{\beta}$ is estimated. We can use the Blogit CML estimator for estimation, as it is consistent under the binomial null hypothesis. We construct a local estimate $\hat{\tau}$ for the object of interest $\tau(0)$ (see Pagan and Ullah, 1999):

$$
\hat{\tau}=\frac{\sum_{i=1}^{N} K\left(\frac{\hat{\Delta}_{i}}{h}\right) z_{i}}{\sum_{i=1}^{N} K\left(\frac{\hat{\Delta}_{i}}{h}\right)}=\sum_{i=1}^{N} w_{n i} z_{i}, \quad w_{n i}=\frac{K\left(\frac{\hat{\Delta}_{i}}{h}\right)}{\sum_{i=1}^{N} K\left(\frac{\hat{\Delta}_{i}}{h}\right)},
$$

The Gaussian function $K\left(\frac{\hat{\Delta}_{i}}{h}\right)=\frac{1}{\sqrt{2 \pi}} \exp \left(-\frac{\left(\hat{\Delta}_{i} / h\right)^{2}}{2}\right)$ is chosen for simplicity. 


\subsection{Asymptotic properties}

Let $f=f(\Delta=0)$ denote the continuous density function of the random variable $\Delta$ at point 0 . The kernel density estimator $\hat{f}$ for $\mathrm{f}$ is

$$
\hat{f}=\sum_{i=1}^{N} \frac{K\left(\frac{\hat{\Delta}_{i}}{h}\right)}{n h}
$$

In addition, rewrite $z_{i}$ as the sum of its conditional expectation $E\left(z_{i} \mid \Delta_{i}\right)=\tau\left(\Delta_{i}\right)$ and an error term $u_{i}$, such that

$$
z_{i}=\tau\left(\Delta_{i}\right)+u_{i}=\left(\Lambda_{i 2}^{\prime} \Delta_{i}\right)^{2}+u_{i}
$$

where $\mathrm{E}\left(u_{i} \mid \Delta_{i}, X_{i}\right)=0$ and $\operatorname{Var}\left(u_{i} \mid \Delta_{i}, X_{i}\right)=\sigma^{2}$.

The estimator $\hat{\tau}$ is a combination of $\hat{f}$ and $z_{i}$

$$
\hat{\tau}=\frac{\sum_{i=1}^{N} \frac{1}{n h} K\left(\frac{\hat{\Delta}_{i}}{h}\right) z_{i}}{\sum_{i=1}^{N} \frac{1}{n h} K\left(\frac{\hat{\Delta}_{i}}{h}\right)}=\frac{1}{\hat{f}} \sum_{i=1}^{N} \frac{1}{n h} K\left(\frac{\hat{\Delta}_{i}}{h}\right) z_{i}=\frac{1}{\hat{f}} \sum_{i=1}^{N} \frac{1}{n h} K\left(\frac{\hat{\Delta}_{i}}{h}\right)\left(\Lambda_{i 2}^{\prime} \Delta_{i}\right)^{2}+u_{i} .
$$

The expectation of $\hat{\tau}$ is

$$
\begin{aligned}
\mathrm{E}(\hat{\tau}) & =E\left(\frac{1}{\hat{f}} \sum_{i=1}^{N} \frac{1}{n h} K\left(\frac{\hat{\Delta}_{i}}{h}\right)\left(\Lambda_{i 2}^{\prime} \Delta_{i}\right)^{2}+\frac{1}{\hat{f}} \sum_{i=1}^{N} \frac{1}{n h} K\left(\frac{\hat{\Delta}_{i}}{h}\right) u_{i}\right) \\
& =\iint \frac{1}{h \hat{f}} K(\nu)\left(\Lambda_{i 2}^{\prime}\right)^{2}(h \nu)^{2} f\left(h \nu, \Lambda_{i 2}\right) h d \nu d \Lambda_{i 2}+\hat{E}\left(u_{i} \mid \hat{\Delta}=0\right), \quad \text { where we replace } \Delta=h \nu \\
& =h^{2} \iint K(\nu)(\nu)^{2}\left(\Lambda_{i 2}^{\prime}\right)^{2} \frac{f\left(\nu, \Lambda_{i 2}\right)}{\hat{f}} d \nu d \Lambda_{i 2} \\
& =h^{2} \mu_{2} E\left[\left(\Lambda_{i 2}^{\prime}\right)^{2} \mid \Delta=0\right], \quad \text { where } \mu_{2}=\int K(\nu)(\nu)^{2} d \nu
\end{aligned}
$$

We therefore obtain a bias

$$
\operatorname{Bias}(\hat{\tau})=\mathrm{E}(\hat{\tau})-\tau(0)=\mathrm{E}(\hat{\tau})=h^{2} \mu_{2} E\left[\left(\Lambda_{i 2}^{\prime}\right)^{2} \mid \Delta=0\right]
$$

that is proportional to $h^{2}$.

To guarantee consistency of the estimator $\hat{\tau}_{n}$, convergence of the mean square error to zero is required. The MSE is equal to $\operatorname{MSE}(\hat{\tau})=\operatorname{Bias}(\hat{\tau})^{2}+\operatorname{Var}(\hat{\tau})$. So the bias for $\tau_{n}$ should decrease to 
zero, as $n$ increases:

$$
h^{2} \longrightarrow 0, \quad \text { as } n \longrightarrow \infty
$$

Besides the convergence condition for bias, we also consider the asymptotic performance of the variance of $\hat{\tau}$. Using a result on the variance of conditional expectations from Pagan and Ullah (1999), we obtain:

$$
\operatorname{Var}(\hat{\tau})=\frac{\sigma^{2}}{n h f} \int K^{2}(\nu) d \nu, \quad \operatorname{Var}(\hat{\tau}) \propto \frac{1}{n h} . \quad \text { If } n \longrightarrow \infty, \quad \frac{1}{n h} \longrightarrow 0 .
$$

To make sure that the MSE converges at the fastest speed, bias ${ }^{2}$ and variance should converge at the same rate: $h^{4} \propto \frac{1}{n h}$. Otherwise, the slower speed dominates the convergence rate. Thus, $h$ is of order $h \propto n^{-\frac{1}{5}}$ and by the central limit theorem,

$$
\sqrt{n h}(\hat{\tau}-E(\hat{\tau})) \stackrel{d}{\longrightarrow} N\left(0, f^{-1} \sigma^{2} \int K^{2}(\nu) d \nu\right)
$$

Here $\sigma^{2}=\operatorname{Var}\left(z_{i}^{2} \mid \Delta=0\right.$ ), with the same definition as in the discrete case (eq. 19). In practice, we standardize $\Delta_{i}$ at first and set bandwidth $h^{\prime}=0.9 n^{-\frac{1}{5}}$. The approximate bias is calculated by $\hat{\mathrm{E}}(\hat{\tau})=\sum_{i=1}^{N} w_{n i}\left(y_{i 2}\left(1-y_{i 2}\right) \hat{\Delta}\right)^{2}, \sigma^{2}$ is replaced by $\hat{\sigma}^{2}=\sum w_{n i}\left(z_{i}-\tau\left(\hat{\Delta}_{i}\right)\right)^{2}$ and $\widehat{\operatorname{Var}}(\hat{\tau})=$ $\frac{\sigma^{2}}{\hat{f}^{2}} \frac{\sum_{i=1}^{N} K^{2}(\nu)}{n^{2} h^{2}}$. Hence, $\frac{\hat{\tau}-\hat{\mathrm{E}}(\hat{\tau})}{\sqrt{\widehat{\operatorname{Var}}(\hat{\tau})}}$ can be used as a $t$-test.

\subsection{Multiple periods}

The test can be extended to multiple time periods. With $T=2$, there is a single moment condition for $E\left(z_{i} \mid \Delta_{i}=0\right)$ that can be tested. For $T>2$, one possibility is to combine $T-1$ such moment conditions into a single test statistic.

In the discrete case, for set $A_{t}=\left\{i: x_{i, t}=x_{i, t+1}\right\}$,

$$
g_{i, t}=\frac{\left(Y_{i, t}-Y_{i, t+1}\right)^{2}-K\left(M_{i, t}-M_{i, t+1}\right)^{2}}{K(K-1)}, \quad t=1, \ldots, T-1
$$

$g_{i, t}$ is empty if $x_{i, t} \neq x_{i, t+1}$. As we derived before, $\mathrm{E}\left(g_{i, t}\right)=0$. 
In matrix form,

$$
g_{i}=\left(\begin{array}{c}
g_{i, 1} \\
\ldots \\
g_{i, T-1}
\end{array}\right), \quad \text { and the sample mean is } \bar{g}_{n}=\left(\begin{array}{c}
\frac{1}{n_{1}} \sum_{i=1}^{n_{1}} g_{i, 1} \\
\ldots \\
\frac{1}{n_{T-1}} \sum_{i=1}^{n_{T-1}} g_{i, T-1}
\end{array}\right) \text {, }
$$

with $n_{t}=\left|A_{t}\right|$, the cardinality of set $A_{t}$. Denote $n=\left(n_{1}, \ldots, n_{T-1}\right)^{\prime}$. To calculate the sample variance $\hat{S}$, we replace off-diagonal elements with pairwise sample covariances and diagonal ones with $g_{t}$ sample variances. A test statistics can be derived

$$
J=\left(\sqrt{n} \circ \bar{g}_{n}\right)^{\prime} \hat{S}^{-1}\left(\sqrt{n} \circ \bar{g}_{n}\right) .
$$

In the continuous case, moment conditions are

$$
g_{i, t}=\frac{K\left(\frac{\hat{\Delta}_{i, t}}{h}\right)\left(z_{i, t}-\tau\left(\hat{\Delta}_{i, t}\right)\right)}{\sum_{i=1}^{N} \frac{1}{n} K\left(\frac{\hat{\Delta}_{i, t}}{h}\right)}, \quad t=1, \ldots, T-1
$$

where $\hat{\Delta}_{i t}=\left(x_{i t}-x_{i, t+1}\right) \hat{\beta}$ and $\tau\left(\hat{\Delta}_{i, t}\right)=\left(y_{i, t+1}\left(1-y_{i, t+1}\right) \hat{\Delta}_{i t}\right)^{2}$. Under the null hypothesis, $\mathrm{E}\left(g_{i, t}\right)=0$.

These moment conditions can be written in matrix form for individual $i=1, \ldots, N$ as :

$$
g_{i}=\left(\begin{array}{c}
g_{i, 1} \\
\ldots \\
g_{i, T-1}
\end{array}\right), \quad \text { and the sample mean is } \bar{g}_{N}=\left(\begin{array}{c}
\frac{1}{N} \sum_{i=1}^{N} g_{i, 1} \\
\ldots \\
\frac{1}{N} \sum_{i=1}^{N} g_{i, T-1}
\end{array}\right)
$$

Let $\hat{S}$ denote the sample variance:

$$
\hat{S}=\frac{1}{N} \sum_{i=1}^{N} g_{i} g_{i}^{\prime}-\bar{g}_{n} \bar{g}_{n}^{\prime}
$$

Since $\bar{g}_{N} \stackrel{p}{\longrightarrow} \mathrm{E}\left(g_{i}\right)=0$, a test statistic is given by

$$
J=N \cdot \bar{g}_{N}^{\prime} \hat{S}^{-1} \bar{g}_{N}=\left(\sqrt{N} \cdot \bar{g}_{N}\right)^{\prime} \hat{S}^{-1}\left(\sqrt{N} \cdot \bar{g}_{N}\right)
$$


Theory: $\quad \sqrt{N} \cdot \bar{g}_{N} \stackrel{d}{\longrightarrow} N(0, S)$ and $\hat{S} \stackrel{p}{\longrightarrow} S$, then $J \stackrel{d}{\longrightarrow} \chi_{T-1}^{2}$.

This chi-square test rejects the binomial distribution assumption at the $\alpha \%$ significance level if $J \geq \chi_{\alpha}^{2}(T-1)$.

\subsection{Simulation study}

We conduct simulation experiments to examine the performance of these tests under two scenarios. In the first setting, explanatory variables are discrete (in fact, there is a single binary regressor, to keep things as simple as possible), while the explanatory variable is continuous in the second. The remaining aspects of the DGP regarding fixed effects, expectation functions and parameters setting are kept the same as those in section 2.3.

-------- Table 2 about here --------

Table 2 presents rejection rates, i.e., the relative number of times that our test rejects the binomial assumption over 1000 replications, when $x$ is discrete. $x_{i t}$ is either 0 or 1 with equal probability. In this case, $\operatorname{Pr}\left(x_{i 1}=x_{i 2}\right)=50 \%$, and on average half of the observations will be in the set $A$ of individuals with the same expectations over time and thus informative for computing the test statistic. As before, simulations are conducted for $T=2,5,10$ and for $K=2,5,10$.

The first row of each sub-panel shows results without overdispersion, i.e., sampling from a binomial DGP applies. In this case, the rejection rates are equivalent to the proportion of type-I errors and ideally should be close to the nominal size of the test, in this case $5 \%$. When $N=100$, the rejection rate is larger than the nominal size (i.e., the test is conservative), especially when $T$ is larger, because convergence in the small sample is slow and the critical value of asymptotic distribution may not be appropriate (Härdle and Mammen 1993). As $N$ increases to 500, we find that rejection rate shrinks towards the expected size. The smallest empirical rejection rate is 4.1 percent, the largest is $15 \%$. If anything, there is a slight tendency to overreject the true assumption, more so in the multivariate version of the test $(T>2)$ than in the scalar version $(T=2)$.

In the current set-up, where we test the validity of a specific model assumption, we would be more worried about under- than overrejection. Also, the power properties are very important in this context. The lower part of each subpanel shows the rejection rates under $H_{0}$ when $H_{0}$ is false, i.e., the binomial model is misspecified. Reassuringly, we find that the test has some power against the 
alternative of rather modest overdispersion (10\%), in particular for $N=500$ and $T=10$, where close to $30 \%$ of wrong null hypotheses are rejected. As the dispersion degree increases, the power of the test also grows, and it reaches $100 \%$ for DGPs where overdispersion degree, the number of observations, and the number of time periods are large.

Table 3 about here

In Table 3, we show the results for the kernel weighted test statistics for continuous regressors. $x_{i t}$ is drawn from a uniform distribution with positive support between -1 and 1 , with mean 0 and variance $1 / 3$. The general patterns regarding type-I errors and power of the tests are mostly similar to those of Table 2. As in Table 2, the power of the test tends to decrease in $K$, for a given overall degree of overdispersion, but this tendency is more uniform in the continuous version of the test. It indicates that the power of the test reacts differently to the two parameters driving overdispersion, and in particular that it is more sensitive to increases in $\phi$ rather than $K$. The combined results from our simulation experiments are reassuring: on one hand, modest amounts of overdispersion cause only minor bias of the Blogit CML estimator; on the other hand, the test we derive has good power properties against medium or high-dispersion alternatives to the binomial assumption.

\section{Application to labor supply}

In this illustrative application, we consider the interplay between fertility and female labor supply in the context of Switzerland, using data from the Swiss Household Panel (SHP) for the years 20122016. The SHP is an ongoing longitudinal survey of households and people living in Switzerland. It collects information on a large range of topics on living conditions, both objective and subjective, including work, fertility and health. We restrict the analysis to women aged 25-45, who participated in the survey at least twice during the five-year period. This gives us a sample of 5,854 person-year observations for 1,712 different women.

The study of female labor supply has a long history (see e.g. Mroz, 1987). Traditionally, the focus has mostly been on the binary participation decision, i.e., the extensive margin, or on annual hours of work, the intensive margin. In the Swiss context, it is more natural to model the work-time percentage, which is a number between 0 and 100\%. These work-time percentages are written into contracts and also advertised in job vacancies. For instance, 60 percent work-time means that the worker works the equivalent of 3 days per week and also is paid $60 \%$ of a full-time salary. In 
practice, the large majority of agreed-upon work-time percentages are multiples of $10 \%$. Figure 3 shows the distribution of work-time percentages in our sample. The relative frequency of zeros is $14.4 \%$, meaning that the estimated participation rate in our sample for this age group is $85.6 \%$, a number very close to the official numbers published by the Federal Statistical Office (BfS, 2016).

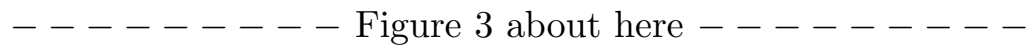

Figure 3 documents one frequently noted "puzzle" of female labor supply in Switzerland: Although the participation rate of women is one of the highest among OECD countries, there is a large prevalence of part-time work, varying from very small amounts to more substantial ones, and the proper full-time rate (defined as, say, working $100 \%$ or $90 \%$ ) is actually not that high (here $21 \%$ ). As a consequence, quite substantial male-female gaps emerge over the life cycle regarding earnings, eligibility for retirement benefits and career development more generally. Clearly, the low work-time percentages for women with children provides a main explanation for this pattern. The Box-plots in Figure 4 show, for our data, that the median work-time percentage drops from 80 percent or higher for those aged 30 or below to 50 percent for women in their early 40s. At the same time, older women are more likely mothers. Around 10 percent of Swiss women remain childless.

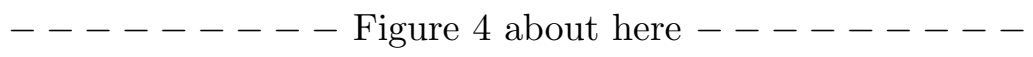

Table 4 provides some descriptive statistics (means and standard deviations) for both the dependent and the explanatory variables used in the estimation. The average work-time percentage is $55 \%$, with a standard deviation of 0.34 . We have re-coded the work-time percentage as a strict multiple of 0.1 , by moving the few intermittent values to the decile below. Hence, we can treat 10 -times the work-time percentage as a binomial variable with outcomes $0,1, \ldots, 10$. Under the binomial assumption, the standard deviation for a fraction with a mean of 0.56 is equal to $\sqrt{0.56(1-0.56) / 10}=0.157$, substantially below the observed standard deviation of 0.346 . Hence, there is evidence of over-dispersion at the marginal level. However, this does not necessarily invalidate the key assumption of the binomial distribution conditional on covariates and individual specific fixed effects. This assumption will be tested.

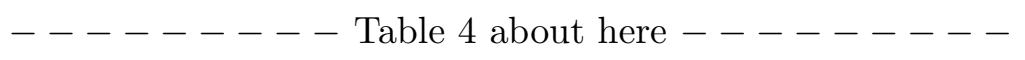

Women have an average age of 36.3 years and 63.1 percent report having at least one child in the year they are surveyed. For 58.4 percent of person-year observations, there is a partner present 
in the household. The health status is captured by a 5-point scale for self assessed health, where 0 means "not well at all" and 4 means "very well". We treat it as a cardinal scale for simplicity, and also abstract from its potential endogeneity to working or having children. Finally, we include information on language region. There is quite a bit of evidence that work-norms differ between the French and the German speaking parts of Switzerland, with some stigma attached to working mothers, in particular during the first years of the child's life, for and full-time work. This stigma seems to be stronger in the German-speaking part of Switzerland (65\% or our sample) but less so in the French-speaking part (29\% of our sample) (see Steinhauer, 2018).

Our final estimation model includes four year dummies, age-squared (the linear age term is dropped; alternatively, one could identify the linear age effect by setting a second year effect equal to zero), indicators for the presence of a child and partner, and the health variable. Since language region is mostly constant over time, it is near-collinear with the fixed effects when applying the Blogit CML or Blogit DV estimators, and we therefore only include its interaction with the child-indicator variable.

As is the case for the binary logit model with fixed effects, DV estimation of the binomial model is subject to the perfect prediction problem (see e.g. Kunz, Staub and Winkelmann, 2018). Outcomes for women, whose work-time percentage is either zero or one in each year are perfectly predicted, meaning that the associated dummy coefficient will tend to minus or plus infinity, respectively. For the Blogit CML, perfectly prediction formally does not arise as the $\alpha_{i}$ 's are not estimated. However, all such observations have mechanically a log-conditional likelihood contribution of zero and thus do not contribute to estimation of $\beta$ either. To use the same estimation sample everywhere, we right away drop all perfectly predicted outcomes, leading to a final sample size of 4,661 person-year observations for the work-time percentage model.

\section{--------- Table 5 about here ---------}

Regression results are given in Table 5. The first column shows the estimated coefficients from the Blogit CML and the second those from the Blogit DV model. The last two columns add corresponding (binary) logit models for the extensive margin model (work yes/no), again using alternatively the CML or DV estimators. Standard errors are clustered at the individual level throughout.

When interpreting magnitudes, we note the recent suggestion by Kemp and Santos Silva (2016) and focus on expected (semi-) elasticities. These can be estimated without knowledge of $\alpha_{i}$ and are 
thus very suitable for our conditional maximum likelihood approach. For the binomial proportion model with $\mathrm{E}\left(y_{i t} \mid x_{i t}, \alpha_{i}\right)=\Lambda_{i t}$, we get

$$
\partial \log \mathrm{E}\left(y_{i t} \mid x_{i t}, \alpha_{i}\right) / \partial x_{i t}=\beta\left(1-\Lambda_{i t}\right)
$$

A good estimator of the overall mean of $\Lambda_{i t}$ is the sample mean of the outcome, $\bar{\Lambda}=\bar{y}=0.55$, so that the CML estimators $\hat{\beta}$ can be multiplied by 0.45 to obtain an estimate of the population average semi-elasticities with respect to changes in the associated covariate.

From columns (1) and (2) of Table 5, we find a large negative association between having a child and the amount of work. The point estimate of the main effect is about -2 , which means that not having a child increases the expected work-time percentage by about 90 percent, a factor of close to two. This effect is highly statistically significant, as are two of the three interaction effects: having a child reduces the work-time percentage more if a partner is present than otherwise, underlining the relevance of pecuniary motives for work, and the need to "make ends meet". The labor supply response of women to having children is about half as large for French speaking women as it is for German speakers, corroborating the social norm results found in the earlier literature (Steinhauer, 2018).

In this application, the Blogit CML and the Blogit DV results are very similar. The DV results are always a bit larger in absolute value, but the difference never exceeds five percent. This resonates with our simulation results, because both $T$ and $K$ are both relatively large. Nevertheless the joint test for the binomial assumption derived in section 3.3 indicates a clear rejection (test value of 37.7 with a $\chi_{0.95}^{2}$ critical value of 9.5). This rejection result due to overdispersion was already foreshadowed, although not logically implied because of the conditional nature of the test, by the high proportion of no work (zero) and full-time work (100\%) as evident in Figure 1. However, we know from the simulation results (Tables 1 and 2) that even with $50 \%$ overdispersion, the bias of the Blogit CML is very small for $K=10$ and $T=5$, a setting similar to the current application. At the same time, the probability of rejecting the wrong $H_{0}$ is very close to 1 (see Table 3 ). On a practical note, the CML estimator can be computed much faster than the DV estimator, by a factor of about 10 in our case. The speed problem of DV models would be exacerbated in applications with more cross-sectional units, to the point where computation of the Blogit DV estimator may become infeasible in the current Stata/R setting.

In the last two columns of Table 5, we allow for a comparison with results from a more conventional 
binary logit extensive margin estimator. A first point to note is that the sample becomes much smaller, since all observations with variation in the positive range only, i.e., percentages between $10 \%$ and $100 \%$, are now coded as "1" and thus become perfectly predicted. Their variation does not contribute to estimation, the usable sample size drops by $3 / 4$, and the standard errors of the estimated coefficients increase accordingly. We had to drop the interaction between speaking Italian and having children, as it could not be estimated in the reduced sample.

The estimated coefficients tend to be substantially larger, but they are not directly comparable. To obtain the implied expected semi-elasticities for the probability of work, coefficients need to be multiplied by the non-participation rate, 0.145 in this case, compared to a factor of 0.45 applicable in the first two columns. Based on the CML estimates, some of the extensive margin semi-elasticities are smaller than the overall semi-elasticities (like the main effect of having a child), and some of them larger (such as self-rated health). In terms of statistical significance, we find that the health and partner coefficients were not statistically in the work-time percentage model, but they are in the participation model. And in terms of point estimate, the interaction between speaking French and having children just offsets the main effect of having at least one child, meaning that there is no difference in participation probabilities for French speaking mothers and non-mothers, although some labor supply responsiveness was found in the work-time percentage model for the combined extensive and intensive margin effect. Finally, in terms of estimation method, and in contrast to the work-time percentage model, the participation model suffers from a massive incidental parameters bias, since the point estimates for the DV estimator exceed those of the CML estimator by fifty percent on average.

\section{Concluding remarks}

Machado (2004) introduced the fixed effects binomial model as a method for proportions or discrete bounded outcomes more generally. However, she did not address the question whether or not the conditional binomial logit maximum likelihood estimator is robust to misspecification. In this paper, we focus on the consequences of overdispersion as it originates, for instance, from neglected unobserved heterogeneity. We show in simulation experiments that the Blogit CML estimator maintains a rather good performance even if the binomial model is misspecified, as long as the length of the panel $T$ is sufficiently large, or the degree of overdispersion is modest.

We then derive a test of the null hypothesis that the binomial assumption is valid against the 
alternative hypothesis of overdispersion. The test computes the variance of within-individual outcome differences. For the subset of observations whose regressors do not change over time, the mean difference is zero (or close to zero if regressors do not differ "too much") and it is possible to compute variances with and without the binomial assumption that do not depend on fixed effects. This is essential, as fixed effects are not estimated by the Blogit CML estimator. Our simulation experiments show that the test has good power properties under the alternative of medium or large degrees of overdispersion. But these are exactly the case when the bias of the Blogit CML estimator becomes noticeable.

Proportions data are ubiquitous in empirical economic research. We study in our empirical application an outcome related to women's work behavior, namely the contracted work-time percentage. In our sample of mid-aged women obtained from the Swiss Household Panel, $65 \%$ of all women report working part-time, i.e. a percentage between $10 \%$ and $90 \%$. The empirical analysis using the fixed effects binomial logit model yields substantially different work-time percentages for mothers and non-mothers. Having a partner makes the effect more pronounced, whereas speaking French reduces it. We show how these coefficients can be interpreted in terms of expected semi-elasticities even if the fixed effects are not estimated. In comparison to the fixed-effects logit estimation for the participation model, much fewer observations are lost in the work-time percentage model due to perfect prediction, contributing to its overall much more precise estimation of the model parameters.

In future work, we will consider alternative estimators that could be pursued if the binomial null hypothesis is rejected. If the logit conditional expectation function is to be kept, a binomial logit correlated random effects model is a possible approach. Such a model would explicitly account for overdispersion, by assuming for instance that unobserved heterogeneity follows a normal distribution with mean depending on the regressors. 


\section{References}

Abrevaya, J. (1997) The equivalence of two estimators of the fixed-effects logit model, Economics Letters 55(1), 41-43.

BfS Bundesamt für Statistik (2016) Arbeitsmarktindikatoren der Schweiz, Neuchâtel, Switzerland.

Chamberlain, G. (1980) Analysis of covariance with qualitative data, Review of Economic Studies, $47,225-238$.

Dean, C.B. (1992) Testing for overdispersion in Poisson and binomial regression models, Journal of the American Statistical Association, 87, 451-457.

Gourieroux, C., A. Monfort and A. Trognon (1984) Pseudo maximum likelihood methods: Theory, Econometrica, 681-700.

Härdle, W. and E. Mammen (1993) Comparing nonparametric versus parametric regression fits, Annals of Statistics, 21(4), 1926-1947

Kemp, G. and J. Santos Silva (2016) Partial effects in fixed-effects models, United Kingdom Stata Users' Group Meetings 2016.

Kunz, J., K. Staub and R. Winkelmann (2018) Predicting fixed effects in panel probit models, York Health, Econometrics and Data Group Working Paper \#18/23.

Machado, M.P. (2004) A consistent estimator for the binomial distribution in the presence of "incidental parameters": an application to patent data, Journal of Econometrics, 119, 73-98.

Maddala, G.S. (1983) Limited-Dependent and Qualitative Variables in Econometrics, Cambridge University Press.

Mroz, T.A. (1987) The Sensitiviy of an Empirical Model of Married Women's Hours of Work to Economic and Statistical Assumptions, Econometrica, 55, 765-799.

Pagan, A. and A. Ullah (1999) Nonparametric Econometrics, Cambridge University Press.

Papke, L.E. and J.M. Wooldridge (1996) Econometric methods for fractional response variables with an application to 401(k) plan participation rates, Journal of Applied Econometrics, 11, 619-632. 
Papke, L.E. and J.M. Wooldridge (2008) Panel data methods for fractional response variables with an application to test pass rates, Journal of Econometrics 145, 121-133.

Ramalho, E.A., J.J.S Ramalho and L.M.S. Coelho (2016) Exponential regression of fractionalresponse fixed-effects models with an application to firm capital structure, Journal of Econometric Methods, 7(1).

Steinhauer, A. (2018) Working Moms, Childlessness, And Female Identity, CEPR Discussion Paper Nr. 12929.

Wooldridge, J.M. (2002) Econometric Analysis of Cross Section and Panel Data, MIT Press. 
Table 1: Simulation Results under the Binomial Distribution

\begin{tabular}{|c|c|c|c|c|c|c|c|c|c|}
\hline \multirow{2}{*}{$\mathrm{N}=100$} & \multicolumn{3}{|c|}{$\mathrm{T}=2$} & \multicolumn{3}{|c|}{$\mathrm{T}=5$} & \multicolumn{3}{|c|}{$\mathrm{T}=10$} \\
\hline & CML & DV & Pooled & CML & DV & Pooled & CML & DV & Pooled \\
\hline \multirow[t]{2}{*}{$\mathrm{K}=2$} & 2.049 & 2.880 & 2.242 & 2.003 & 2.280 & 1.986 & 2.006 & 2.134 & 1.877 \\
\hline & $(0.419)$ & $(0.621)$ & $(0.255)$ & $(0.178)$ & $(0.211)$ & $(0.145)$ & $(0.118)$ & $(0.128)$ & $(0.101)$ \\
\hline \multirow[t]{2}{*}{$\mathrm{K}=5$} & 2.012 & 2.279 & 2.243 & 2.000 & 2.101 & 1.990 & 2.002 & 2.051 & 1.872 \\
\hline & $(0.233)$ & $(0.272)$ & $(0.190)$ & (0.111) & $(0.118)$ & $(0.104)$ & $(0.073)$ & $(0.075)$ & $(0.076)$ \\
\hline \multirow[t]{2}{*}{$\mathrm{K}=10$} & 2.005 & 2.129 & 2.241 & 2.001 & 2.050 & 1.989 & 2.000 & 2.025 & 1.871 \\
\hline & $(0.157)$ & $(0.169)$ & $(0.153)$ & $(0.078)$ & $(0.081)$ & $(0.091)$ & $(0.052)$ & $(0.052)$ & $(0.063)$ \\
\hline \multirow[t]{2}{*}{$\mathrm{N}=500$} & \multicolumn{3}{|c|}{$\mathrm{T}=2$} & \multicolumn{3}{|c|}{$\mathrm{T}=5$} & \multicolumn{3}{|c|}{$\mathrm{T}=10$} \\
\hline & CML & DV & Pooled & CML & DV & Pooled & CML & DV & Pooled \\
\hline \multirow[t]{2}{*}{$\mathrm{K}=2$} & 2.013 & 2.826 & 2.234 & 2.002 & 2.278 & 1.987 & 2.002 & 2.130 & 1.870 \\
\hline & $(0.170)$ & $(0.254)$ & $(0.112)$ & $(0.082)$ & $(0.097)$ & $(0.068)$ & $(0.053)$ & $(0.057)$ & $(0.046)$ \\
\hline \multirow[t]{2}{*}{$\mathrm{K}=5$} & 2.003 & 2.268 & 2.232 & 2.001 & 2.102 & 1.986 & 2.000 & 2.049 & 1.867 \\
\hline & $(0.102)$ & $(0.119)$ & $(0.078)$ & $(0.051)$ & $(0.054)$ & $(0.050)$ & $(0.033)$ & $(0.034)$ & (0.033) \\
\hline \multirow[t]{2}{*}{$\mathrm{K}=10$} & 1.997 & 2.121 & 2.235 & 1.999 & 2.049 & 1.984 & 2.000 & 2.024 & 1.867 \\
\hline & $(0.071)$ & $(0.076)$ & $(0.067)$ & $(0.034)$ & $(0.035)$ & $(0.042)$ & $(0.023)$ & $(0.023)$ & $(0.029)$ \\
\hline
\end{tabular}

Results for 1000 Monte Carlo replications; Standard deviations in parentheses. The number of observation in each period is 100 in the upper part of the table and 500 in the second half. $x_{i t} \sim U[-1,1] . \beta_{1}=2$ and $\alpha_{i}=\sqrt{T} \bar{x}_{i}+N(0,1)$. Outcomes $y_{i t}$ follows the binomial distribution with $p_{i t}=\frac{\exp \left(x_{i t} \beta_{1}+\alpha_{i}\right)}{1+\exp \left(x_{i t} \beta_{1}+\alpha_{i}\right)}$. 
Table 2: Simulation Results for Rejection Rates when $x$ is Discrete

\begin{tabular}{cc|ccccc|ccc}
\hline \hline $\mathrm{N}=100$ & Dispersion & $\mathrm{T}=2$ & $\mathrm{~T}=5$ & $\mathrm{~T}=10$ & $\mathrm{~N}=500$ & Dispersion & $\mathrm{T}=2$ & $\mathrm{~T}=5$ & $\mathrm{~T}=10$ \\
\hline$K=2$ & $0 \%$ & 0.062 & 0.150 & 0.447 & $K=2$ & $0 \%$ & 0.054 & 0.071 & 0.129 \\
& $10 \%$ & 0.080 & 0.216 & 0.477 & & $10 \%$ & 0.143 & 0.222 & 0.373 \\
& $50 \%$ & 0.502 & 0.797 & 0.743 & & $50 \%$ & 0.992 & 1 & 1 \\
\hline$K=5$ & $0 \%$ & 0.056 & 0.175 & 0.444 & $K=5$ & $0 \%$ & 0.041 & 0.078 & 0.134 \\
& $10 \%$ & 0.044 & 0.186 & 0.429 & & $10 \%$ & 0.094 & 0.150 & 0.250 \\
& $50 \%$ & 0.225 & 0.501 & 0.737 & & $50 \%$ & 0.861 & 0.995 & 1.000 \\
& $100 \%$ & 0.603 & 0.899 & 0.958 & & $100 \%$ & 1 & 1 & 1 \\
& $200 \%$ & 0.938 & 0.992 & 0.968 & & $200 \%$ & 1 & 1 & 1 \\
\hline$K=10$ & $0 \%$ & 0.052 & 0.169 & 0.426 & $K=10$ & $0 \%$ & 0.059 & 0.059 & 0.149 \\
& $10 \%$ & 0.052 & 0.197 & 0.422 & & $10 \%$ & 0.083 & 0.130 & 0.211 \\
& $50 \%$ & 0.204 & 0.428 & 0.700 & & $50 \%$ & 0.813 & 0.991 & 1.000 \\
& $100 \%$ & 0.492 & 0.823 & 0.941 & & $100 \%$ & 1 & 1 & 1 \\
& $200 \%$ & 0.869 & 0.993 & 0.992 & & $200 \%$ & 1 & 1 & 1 \\
\hline \hline
\end{tabular}

Notes: $x_{i t}$ is binary variable with $50 \%$ probability equal to 0 or 1 . The rest DGP is same as in Table 3 ; The null hypothesis is that of binomial dispersion.

Table 3: Simulation Results for Rejection Rates when $x$ is Continuous

\begin{tabular}{cc|ccccc|ccc}
\hline \hline $\mathrm{N}=100$ & Dispersion & $\mathrm{T}=2$ & $\mathrm{~T}=5$ & $\mathrm{~T}=10$ & $\mathrm{~N}=500$ & Dispersion & $\mathrm{T}=2$ & $\mathrm{~T}=5$ & $\mathrm{~T}=10$ \\
\hline$K=2$ & $0 \%$ & 0.053 & 0.063 & 0.097 & $K=2$ & $0 \%$ & 0.062 & 0.047 & 0.056 \\
& $10 \%$ & 0.070 & 0.095 & 0.185 & & $10 \%$ & 0.138 & 0.223 & 0.394 \\
& $50 \%$ & 0.424 & 0.684 & 0.899 & & $50 \%$ & 0.948 & 1 & 1 \\
\hline$K=5$ & $0 \%$ & 0.05 & 0.053 & 0.078 & $K=5$ & $0 \%$ & 0.042 & 0.05 & 0.057 \\
& $10 \%$ & 0.044 & 0.063 & 0.110 & & $10 \%$ & 0.083 & 0.105 & 0.190 \\
& $50 \%$ & 0.239 & 0.512 & 0.805 & & $50 \%$ & 0.772 & 0.992 & 1 \\
& $100 \%$ & 0.608 & 0.958 & 0.999 & & $100 \%$ & 0.996 & 1 & 1 \\
& $200 \%$ & 0.927 & 0.991 & 0.995 & & $200 \%$ & 1 & 1 & 1 \\
\hline$K=10$ & $0 \%$ & 0.041 & 0.062 & 0.091 & $K=10$ & $0 \%$ & 0.052 & 0.056 & 0.043 \\
& $10 \%$ & 0.041 & 0.057 & 0.086 & & $10 \%$ & 0.073 & 0.094 & 0.179 \\
& $50 \%$ & 0.190 & 0.402 & 0.673 & & $50 \%$ & 0.677 & 0.988 & 1 \\
& $100 \%$ & 0.482 & 0.908 & 0.997 & & $100 \%$ & 0.991 & 1 & 1 \\
& $200 \%$ & 0.873 & 0.999 & 0.999 & & $200 \%$ & 1 & 1 & 1 \\
\hline \hline
\end{tabular}

Note: Results for 1000 Monte Carlo replications; Standard deviations in parentheses. In each period, the number of observation is 100. $x_{i t} \sim U[-1,1] . \beta_{1}=2$ and $\alpha_{i}=\sqrt{T} \bar{x}_{i}+N(0,1)$. Overdispersion factor $\frac{k-1}{\phi+1}$ represents dispersion degree of variance. Overdispersion degree $0 \%$ is generated by binomial distribution and postive dispersion degree is generated by a beta-binomial DGP. 
Table 4. Descriptive statistics $(N T=5,854)$

\begin{tabular}{lcc}
\hline \hline & mean & std. dev. \\
\hline Work-time percentage & 0.557 & 0.346 \\
Age & 36.30 & 6.01 \\
& & \\
Children (yes=1) & 0.631 & 0.482 \\
Partner (yes=1) & 0.584 & 0.492 \\
& & \\
Self-rated health & 3.114 & 0.610 \\
Years of schooling & 14.43 & 3.09 \\
& & \\
French speaking (yes=1) & 0.293 & 0.455 \\
Italian speaking (yes=1) & 0.043 & 0.204 \\
\hline \hline
\end{tabular}

Source: Swiss Household Panel 2012-2016, own calculations. 
Table 5. Determinants of female labor supply (SHP 2012-2016)

\begin{tabular}{lcccc}
\hline \hline & \multicolumn{2}{c}{ Work-time Percentage } & \multicolumn{2}{c}{ Work (yes/no) } \\
& Blogit CML & Blogit DV & Logit CML & Logit DV \\
\hline Age squared & -0.001 & -0.001 & 0.005 & 0.007 \\
& $(0.002)$ & $(0.002)$ & $(0.005)$ & $(0.008)$ \\
Self-rated health & 0.071 & 0.073 & 0.448 & 0.642 \\
& $(0.038)$ & $(0.039)$ & $(0.153)$ & $(0.220)$ \\
Partner (yes=1) & 0.322 & 0.333 & 1.371 & 1.927 \\
& $(0.253)$ & $(0.260)$ & $(0.658)$ & $(0.952)$ \\
Children (yes=1) & -2.097 & -2.160 & -1.975 & -2.771 \\
& $(0.276)$ & $(0.286)$ & $(0.956)$ & $(1.381)$ \\
Children $\times$ Partner & -0.824 & -0.848 & -2.216 & -3.188 \\
& $(0.260)$ & $(0.268)$ & $(1.056)$ & $(1.510)$ \\
Children $\times$ French & 1.152 & 1.194 & 1.876 & 2.777 \\
& $(0.405)$ & $(0.418)$ & $(1.089)$ & $(1.619)$ \\
Children $\times$ Italian & -0.247 & -0.266 & & \\
& $(0.720)$ & $(0.754)$ & & \\
Year 2013 & 0.143 & 0.147 & -0.018 & -0.018 \\
& $(0.147)$ & $(0.151)$ & $(0.465)$ & $(0.647)$ \\
Year 2014 & 0.246 & 0.253 & -0.147 & -0.265 \\
Year 2015 & $(0.278)$ & $(0.285)$ & $(0.820)$ & $(1.143)$ \\
Year 2016 & 0.338 & 0.347 & -0.247 & -0.408 \\
& $(0.412)$ & $(0.423)$ & $(1.205)$ & $(1.677)$ \\
Number of person-years & 0.387 & 0.397 & -0.449 & -0.717 \\
Number of persons & 1,661 & 4,661 & 1,071 & 1,071 \\
Log pseudolikelihood & $-23,183.6$ & $-1,838.3$ & -358.8 & -595.9 \\
Fixed effects & yes & yes & yes & yes \\
\hline \hline
\end{tabular}


Figure 1: Relative Bias By Dispersion Degree (N=100)
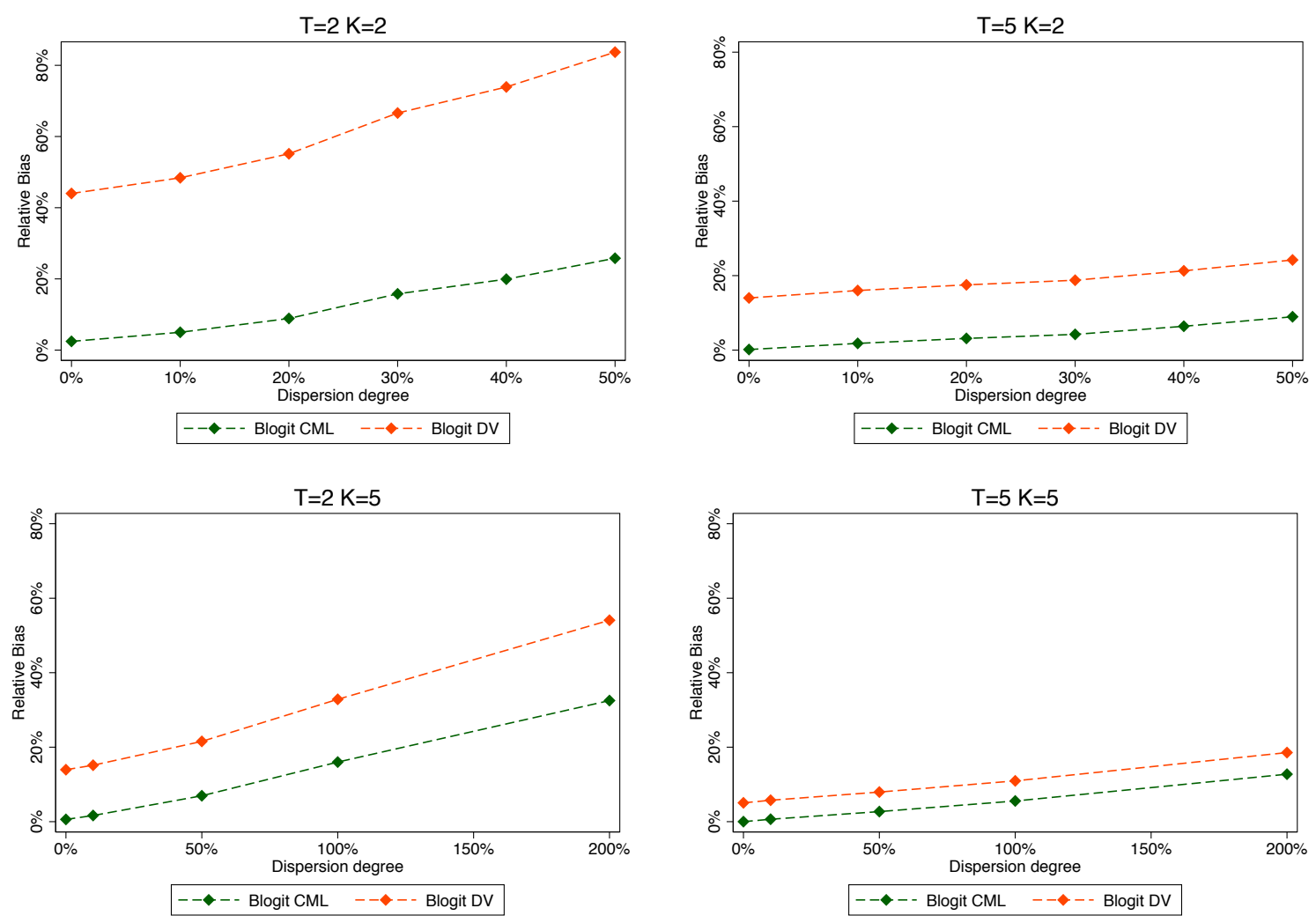
Figure 2: Relative BIAS By Dispersion Degree $(\mathrm{N}=500)$
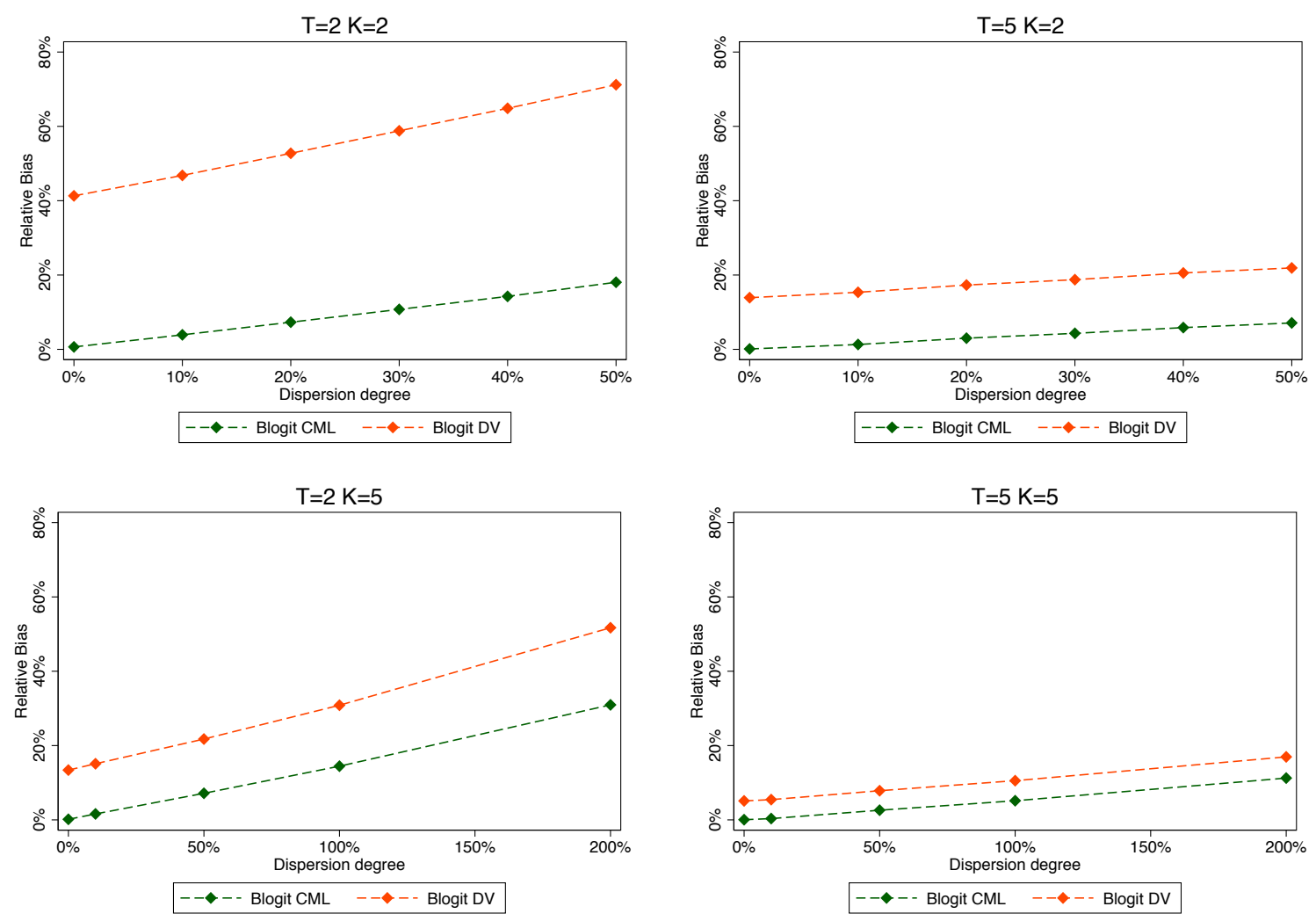
Figure 3: Distribution of Work-time PERCEntage in 2016

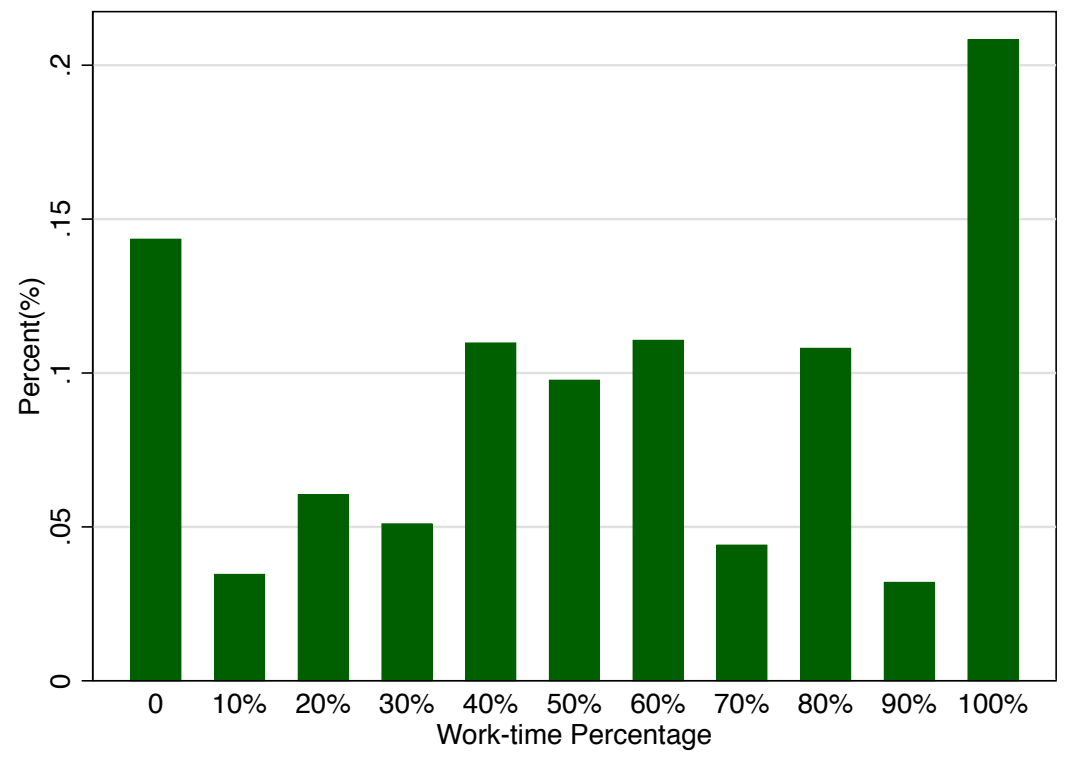


Figure 4: WoRK-TIME PERCENTAGE AND MOTHERHOOD BY AGE IN 2016

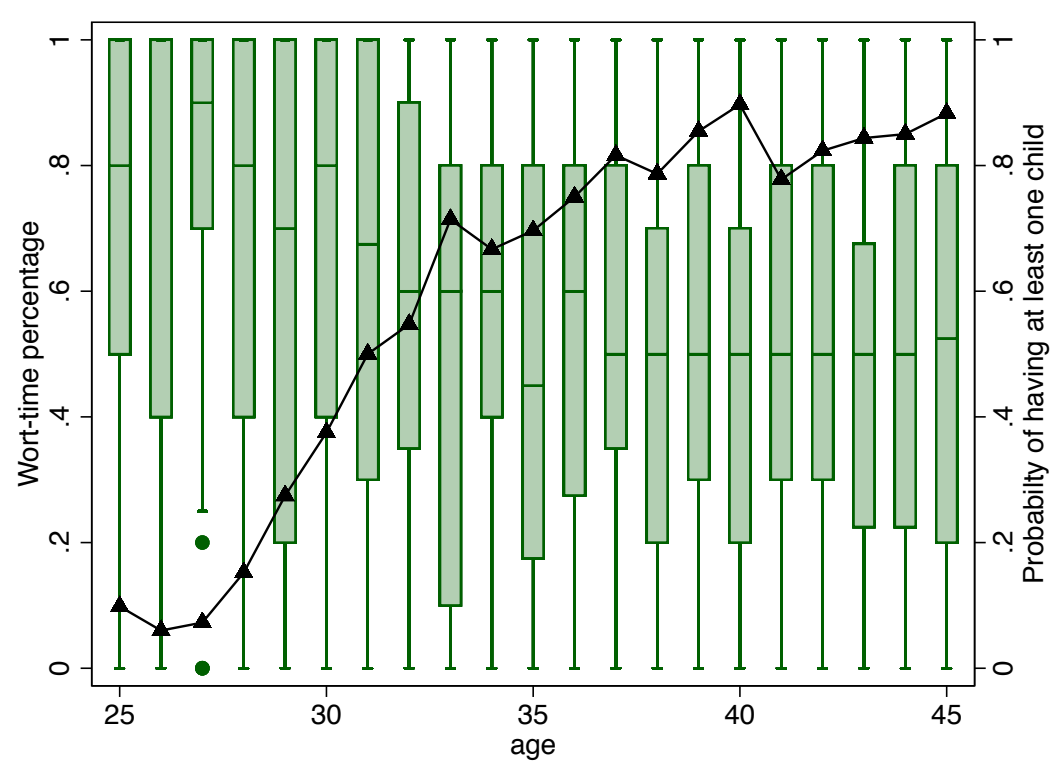


Appendix A: Simulation Results $\mathrm{N}=100$

\begin{tabular}{|c|c|c|c|c|c|c|c|c|c|c|}
\hline \multirow{2}{*}{$\mathrm{N}=100$} & \multirow[t]{2}{*}{ Dispersion } & \multicolumn{3}{|c|}{$\mathrm{T}=2$} & \multicolumn{3}{|c|}{$\mathrm{T}=5$} & \multicolumn{3}{|c|}{$\mathrm{T}=10$} \\
\hline & & CML & DV & Pooled & CML & DV & Pooled & CML & DV & Pooled \\
\hline \multirow[t]{4}{*}{$\mathrm{K}=2$} & $0 \%$ & 2.049 & 2.880 & 2.242 & 2.003 & 2.280 & 1.986 & 2.006 & 2.134 & 1.877 \\
\hline & \multirow[b]{2}{*}{$10 \%$} & & & & & & & & & \\
\hline & & $\begin{array}{c}2.100 \\
(0.419)\end{array}$ & $\begin{array}{c}2.968 \\
(0.632)\end{array}$ & $\begin{array}{c}2.225 \\
(0.263)\end{array}$ & $\begin{array}{c}2.036 \\
(0.192)\end{array}$ & $\begin{array}{c}2.320 \\
(0.227)\end{array}$ & $\begin{array}{c}1.988 \\
(0.156)\end{array}$ & $\begin{array}{c}2.010 \\
(0.121)\end{array}$ & $\begin{array}{c}2.138 \\
(0.131)\end{array}$ & $\begin{array}{c}1.876 \\
(0.105)\end{array}$ \\
\hline & $50 \%$ & $\begin{array}{c}2.516 \\
(0.822)\end{array}$ & $\begin{array}{c}3.674 \\
(1.283)\end{array}$ & $\begin{array}{c}1.898 \\
(0.399)\end{array}$ & $\begin{array}{c}2.179 \\
(0.360)\end{array}$ & $\begin{array}{c}2.484 \\
(0.429)\end{array}$ & $\begin{array}{c}1.908 \\
(0.279)\end{array}$ & $\begin{array}{c}2.073 \\
(0.246)\end{array}$ & $\begin{array}{c}2.204 \\
(0.266)\end{array}$ & $\begin{array}{c}1.937 \\
(0.222)\end{array}$ \\
\hline \multirow{11}{*}{$\mathrm{K}=5$} & \multirow[t]{2}{*}{ Dispersion } & \multicolumn{3}{|c|}{$\mathrm{T}=2$} & \multicolumn{3}{|c|}{$\mathrm{T}=5$} & \multicolumn{3}{|c|}{$\mathrm{T}=10$} \\
\hline & & CML & DV & Pooled & CML & DV & Pooled & CML & DV & Pooled \\
\hline & $0 \%$ & $\begin{array}{l}2.012 \\
(0.223)\end{array}$ & $\begin{array}{l}2.279 \\
(0.279)\end{array}$ & 2.243 & $\begin{array}{l}2.000 \\
(0.111)\end{array}$ & $\begin{array}{c}2.101 \\
(0.118)\end{array}$ & 1.990 & $\begin{array}{l}2.002 \\
(0.073)\end{array}$ & $\begin{array}{l}2.051 \\
(0.075)\end{array}$ & 1.872 \\
\hline & \multirow[t]{2}{*}{$10 \%$} & 2.033 & 2.303 & 2.239 & 2.013 & 2.115 & 1.990 & 2.010 & 2.059 & 1.875 \\
\hline & & $(0.252)$ & $(0.295)$ & $(0.184)$ & $(0.120)$ & $(0.128)$ & $(0.115)$ & $(0.079)$ & $(0.081)$ & $(0.078)$ \\
\hline & \multirow[t]{2}{*}{$50 \%$} & 2.139 & 2.431 & 2.205 & 2.054 & 2.159 & 1.971 & 2.032 & 2.081 & 1.882 \\
\hline & & $(0.300)$ & $(0.354)$ & $(0.214)$ & (0.139) & $(0.149)$ & $(0.122)$ & $(0.086)$ & (0.088) & (0.083) \\
\hline & \multirow[t]{2}{*}{$100 \%$} & 2.320 & 2.657 & 2.140 & 2.111 & 2.219 & 1.958 & 2.052 & 2.102 & 1.898 \\
\hline & & $(0.402)$ & $(0.485)$ & $(0.253)$ & $(0.176)$ & (0.188) & $(0.147)$ & $(0.116)$ & $(0.119)$ & $(0.104)$ \\
\hline & \multirow[t]{2}{*}{$200 \%$} & 2.721 & 3.167 & 1.887 & 2.231 & 2.345 & 1.891 & 2.115 & 2.167 & 1.948 \\
\hline & & $(0.736)$ & $(0.910)$ & $(0.354)$ & $(0.306)$ & $(0.326)$ & $(0.246)$ & $(0.208)$ & $(0.214)$ & $(0.186)$ \\
\hline \multirow{12}{*}{$\mathrm{K}=10$} & \multirow[t]{2}{*}{ Dispersion } & \multicolumn{3}{|c|}{$\mathrm{T}=2$} & \multicolumn{3}{|c|}{$\mathrm{T}=5$} & \multicolumn{3}{|c|}{$\mathrm{T}=10$} \\
\hline & & CML & DV & Pooled & CML & DV & Pooled & CML & DV & Pooled \\
\hline & \multirow[t]{2}{*}{$0 \%$} & 2.005 & 2.129 & 2.241 & 2.001 & 2.050 & 1.989 & 2.000 & 2.025 & 1.871 \\
\hline & & $(0.157)$ & (0.169) & $(0.153)$ & (0.078) & $(0.081)$ & (0.091) & $(0.052)$ & $(0.052)$ & $(0.063)$ \\
\hline & \multirow[t]{2}{*}{$10 \%$} & 2.017 & 2.142 & 2.237 & 2.004 & 2.053 & 1.986 & 2.002 & 2.026 & 1.868 \\
\hline & & $(0.174)$ & $(0.187)$ & $(0.158)$ & $(0.082)$ & $(0.084)$ & $(0.091)$ & $(0.055)$ & $(0.055)$ & $(0.063)$ \\
\hline & \multirow[t]{2}{*}{$50 \%$} & 2.065 & 2.195 & 2.241 & 2.026 & 2.076 & 1.984 & 2.012 & 2.036 & 1.869 \\
\hline & & $(0.202)$ & $(0.219)$ & $(0.175)$ & (0.098) & $(0.101)$ & $(0.100)$ & $(0.064)$ & $(0.065)$ & $(0.072)$ \\
\hline & \multirow[t]{2}{*}{$100 \%$} & 2.139 & 2.275 & 2.214 & 2.051 & 2.102 & 1.979 & 2.025 & 2.049 & 1.876 \\
\hline & & $(0.239)$ & $(0.260)$ & $(0.183)$ & $(0.109)$ & $(0.113)$ & $(0.106)$ & $(0.074)$ & $(0.075)$ & $(0.076)$ \\
\hline & \multirow[t]{2}{*}{$200 \%$} & 2.293 & 2.443 & 2.146 & 2.105 & 2.157 & 1.963 & 2.049 & 2.073 & 1.892 \\
\hline & & $(0.325)$ & $(0.354)$ & $(0.215)$ & $(0.148)$ & $(0.153)$ & $(0.124)$ & $(0.095)$ & $(0.096)$ & $(0.087)$ \\
\hline
\end{tabular}

Results for 1000 Monte Carlo replications; Standard deviations in parentheses. In each period, the number of observation is 100. $x_{i t} \sim U[-1,1] . \beta_{1}=2$ and $\alpha_{i}=\sqrt{T} \bar{x}_{i}+N(0,1)$. Overdispersion factor $\frac{k-1}{\phi+1}$ represents dispersion degree of variance. Overdispersion degree $0 \%$ is generated by binomial distribution and postive dispersion degree is generated by a beta-binomial DGP. 
Appendix B: Simulation Results $\mathrm{N}=500$

\begin{tabular}{|c|c|c|c|c|c|c|c|c|c|c|}
\hline \multirow{2}{*}{$\mathrm{N}=500$} & \multirow[t]{2}{*}{ Dispersion } & \multicolumn{3}{|c|}{$\mathrm{T}=2$} & \multicolumn{3}{|c|}{$\mathrm{T}=5$} & \multicolumn{3}{|c|}{$\mathrm{T}=10$} \\
\hline & & CML & DV & Pooled & CML & DV & Pooled & CML & DV & Pooled \\
\hline \multirow[t]{5}{*}{$\mathrm{K}=2$} & \multirow[t]{2}{*}{$0 \%$} & 2.013 & 2.826 & 2.234 & 2.002 & 2.278 & 1.987 & 2.002 & 2.130 & 1.870 \\
\hline & & $(0.170)$ & $(0.254)$ & $(0.112)$ & $(0.082)$ & $(0.097)$ & $(0.068)$ & $(0.053)$ & $(0.057)$ & $(0.046)$ \\
\hline & \multirow[t]{2}{*}{$10 \%$} & 2.078 & 2.936 & 2.214 & 2.026 & 2.307 & 1.975 & 2.011 & 2.139 & 1.870 \\
\hline & & $(0.195)$ & $(0.293)$ & $(0.120)$ & $(0.084)$ & $(0.099)$ & $(0.070)$ & $(0.058)$ & $(0.062)$ & $(0.050)$ \\
\hline & $50 \%$ & $\begin{array}{c}2.361 \\
(0.311)\end{array}$ & $\begin{array}{c}3.424 \\
(0.489)\end{array}$ & $\begin{array}{c}1.849 \\
(0.175)\end{array}$ & $\begin{array}{c}2.142 \\
(0.151)\end{array}$ & $\begin{array}{c}2.438 \\
(0.180)\end{array}$ & $\begin{array}{c}1.881 \\
(0.120)\end{array}$ & $\begin{array}{c}2.062 \\
(0.102)\end{array}$ & $\begin{array}{c}2.191 \\
(0.110)\end{array}$ & $\begin{array}{c}1.926 \\
(0.092)\end{array}$ \\
\hline \multirow{12}{*}{$\mathrm{K}=5$} & \multirow[t]{2}{*}{ Dispersion } & \multicolumn{3}{|c|}{$\mathrm{T}=2$} & \multicolumn{3}{|c|}{$\mathrm{T}=5$} & \multicolumn{3}{|c|}{$\mathrm{T}=10$} \\
\hline & & CML & DV & Pooled & CML & DV & Pooled & CML & DV & Pooled \\
\hline & \multirow[t]{2}{*}{$0 \%$} & 2.003 & 2.268 & 2.232 & 2.001 & 2.102 & 1.986 & 2.000 & 2.049 & 1.867 \\
\hline & & $(0.102)$ & $(0.119)$ & $(0.078)$ & $(0.051)$ & $(0.054)$ & $(0.050)$ & $(0.033)$ & $(0.034)$ & $(0.033)$ \\
\hline & \multirow[t]{2}{*}{$10 \%$} & 2.032 & 2.302 & 2.237 & 2.007 & 2.109 & 1.984 & 2.006 & 2.055 & 1.868 \\
\hline & & $(0.108)$ & $(0.126)$ & $(0.088)$ & $(0.052)$ & $(0.055)$ & $(0.051)$ & $(0.035)$ & $(0.036)$ & $(0.034)$ \\
\hline & \multirow[t]{2}{*}{$50 \%$} & 2.143 & 2.435 & 2.204 & 2.052 & 2.157 & 1.973 & 2.023 & 2.073 & 1.871 \\
\hline & & $(0.132)$ & $(0.156)$ & $(0.090)$ & $(0.063)$ & $(0.068)$ & $(0.055)$ & $(0.041)$ & $(0.043)$ & $(0.040)$ \\
\hline & \multirow[t]{2}{*}{$100 \%$} & 2.289 & 2.617 & 2.121 & 2.103 & 2.211 & 1.951 & 2.050 & 2.100 & 1.895 \\
\hline & & $(0.168)$ & $(0.203)$ & $(0.107)$ & $(0.080)$ & $(0.085)$ & $(0.063)$ & $(0.049)$ & $(0.051)$ & $(0.046)$ \\
\hline & \multirow[t]{2}{*}{$200 \%$} & 2.619 & 3.034 & 1.852 & 2.225 & 2.339 & 1.882 & 2.105 & 2.156 & 1.932 \\
\hline & & $(0.292)$ & $(0.363)$ & $(0.152)$ & $(0.137)$ & $(0.146)$ & $(0.109)$ & $(0.093)$ & $(0.095)$ & $(0.083)$ \\
\hline \multirow{12}{*}{$\mathrm{K}=10$} & \multirow[t]{2}{*}{ Dispersion } & \multicolumn{3}{|c|}{$\mathrm{T}=2$} & \multicolumn{3}{|c|}{$\mathrm{T}=5$} & \multicolumn{3}{|c|}{$\mathrm{T}=10$} \\
\hline & & CML & DV & Pooled & CML & DV & Pooled & CML & $\mathrm{DV}$ & Pooled \\
\hline & \multirow[t]{2}{*}{$0 \%$} & 1.997 & 2.121 & 2.235 & 1.999 & 2.049 & 1.984 & 2.000 & 2.024 & 1.867 \\
\hline & & $(0.071)$ & $(0.076)$ & $(0.067)$ & $(0.034)$ & $(0.035)$ & $(0.042)$ & $(0.023)$ & $(0.023)$ & $(0.029)$ \\
\hline & \multirow[t]{2}{*}{$10 \%$} & 2.014 & 2.140 & 2.233 & 2.003 & 2.053 & 1.986 & 2.002 & 2.026 & 1.869 \\
\hline & & $(0.075)$ & $(0.081)$ & $(0.073)$ & $(0.038)$ & $(0.039)$ & $(0.043)$ & $(0.025)$ & $(0.025)$ & $(0.031)$ \\
\hline & \multirow[t]{2}{*}{$50 \%$} & 2.066 & 2.195 & 2.228 & 2.023 & 2.073 & 1.980 & 2.013 & 2.037 & 1.868 \\
\hline & & $(0.092)$ & $(0.099)$ & $(0.076)$ & $(0.043)$ & $(0.044)$ & $(0.044)$ & $(0.029)$ & $(0.029)$ & $(0.033)$ \\
\hline & \multirow[t]{2}{*}{$100 \%$} & 2.134 & 2.269 & 2.206 & 2.050 & 2.100 & 1.974 & 2.024 & 2.048 & 1.871 \\
\hline & & $(0.108)$ & $(0.117)$ & $(0.083)$ & $(0.050)$ & $(0.051)$ & $(0.048)$ & $(0.035)$ & $(0.035)$ & $(0.035)$ \\
\hline & $200 \%$ & 2.293 & 2.443 & 2.146 & 2.105 & 2.157 & 1.963 & 2.049 & 2.074 & 1.890 \\
\hline & & $(0.325)$ & $(0.354)$ & $(0.215)$ & $(0.148)$ & $(0.153)$ & $(0.124)$ & $(0.043)$ & $(0.043)$ & $(0.040)$ \\
\hline
\end{tabular}

Notes: see Appendix A. 